This document is confidential and is proprietary to the American Chemical Society and its authors. Do not copy or disclose without written permission. If you have received this item in error, notify the sender and delete all copies.

\title{
Photophysical Heavy-Atom Effect in Iodinated Metallocorroles: Spin-Orbit Coupling and Density of States
}

\begin{tabular}{|r|l|}
\hline Journal: & The Journal of Physical Chemistry \\
\hline Manuscript ID & jp-2018-053112.R1 \\
\hline Danuscript Type: & Article \\
\hline Complete List of Authors: & $\begin{array}{l}\text { Pomarico, Enrico; Ecole polytechnique federale de Lausanne Faculte des } \\
\text { sciences de base, } \\
\text { Pospisil, Petr; J. Heyrovsky Institute of Physical Chemistry, } \\
\text { Bouduban, Marine; Ecole Polytechnique Federale de Lausanne, Institute of } \\
\text { Chemical Sciences and Engineering } \\
\text { Vestfrid, Jenya; Israel Institute of Technology, Shulich Faculty of Chemistry } \\
\text { Technion } \\
\text { Gross, Zeev; Technion, Israel Institute of Technology, Shulich Faculty of } \\
\text { Chemistry } \\
\text { Zalis, Stanislav; J. Heyrovsky Institute of Physical Chemistry, } \\
\text { Chergui, Majed; Ecole Polytechnique Fédérale de Lausanne, Lab. of } \\
\text { Ultrafast Spectroscopy } \\
\text { Vlcek, Antonin; Queen Mary, University of London, SBCS }\end{array}$ \\
\hline
\end{tabular}

\section{SCHOLARONE ${ }^{m}$}

Manuscripts 


\begin{abstract}
Excited-state dynamics and electronic structures of Al and Ga corrole complexes were studied as a function of the number of $\beta$-pyrrole iodine substituents. Using spectrally broad-band femtosecond-resolved fluorescence upconversion, we determined the kinetics of the Soret fluorescence decay, the concomitant rise and subsequent decay of the Q-band fluorescence, as well as of the accompanying vibrational relaxation. lodination was found to accelerate all involved processes. The time constant of the internal conversion from the Soret to the $Q$ states decreases from 320-540 to 70-185 fs upon iodination. Vibrational relaxation then occurs with ca. 15 and 0.36-1.4 ps lifetime for iodine-free and iodinated complexes, respectively. Intersystem crossing to the lowest triplet is accelerated up to $200 \times$, from nanoseconds to $15-24$ ps; and its rate correlates with the iodine $p(\pi)$ participation in the corrole $\pi$ system and the spin-orbit coupling (SOC) strength. TDDFT calculations with explicit SOC show that iodination introduces a manifold of low-lying singlet and triplet iodine $\rightarrow$ corrole charge-transfer (CT) states. These states affect the photophysics by: (i) providing a relaxation cascade for the Soret $\rightarrow Q$ internal conversion and cooling; and (ii) opening new SOC pathways whereby CT triplet character is admixed into both $\mathrm{Q}$ singlet excited states. In addition, SOC between the higher $Q$ singlet and the Soret triplet is enhanced as the iodine participation in frontier corrole $\pi$ orbitals increases. Our observations that iodination of the chromophore periphery affects the whole photocycle by changing the electronic structure, spin-orbit coupling, and the density of states rationalize the "heavy-atom effect", and have implications for controlling excited-state dynamics in a range of triplet photosensitizers.
\end{abstract}




\section{Introduction}

Corroles and their metal complexes ${ }^{1,2,3}$ exhibit very rich photophysical, $4,5,6,7,8,9,10,11$ photochemical, $^{12}$ and redox $^{13}$ behaviors that are tunable and controllable by varying substituents on the molecular periphery, as well as the central metal atom. ${ }^{14}$ Corroles are intensely studied for their catalytic (e.g., hydrogen evolution, ${ }^{15,16}$ oxygen reduction, ${ }^{17,18}$ water oxidation, $^{19}$ peroxynitrite decomposition, ${ }^{20}$ epoxidation, ${ }^{21}$ etc.) and sensor $^{22,23}$ properties. Importantly, corroles and their metal complexes are excellent agents for photodynamic therapy and inhibition, ${ }^{24}$ cancer-targeted imaging and tumor elimination, ${ }^{25,26,27,28,29}$ as well as solarcell $^{30,31}$ and singlet-oxygen ${ }^{22,24,32,33,34,35}$ photosensitizers. It is also possible to envisage corroles as active components of electron- and energy transfer supramolecular assemblies, although this opportunity has been explored less. ${ }^{31}$ They also exhibit pronounced 2-photon absorption and high nonlinear optical coefficients, opening interesting possibilities for optoelectronic applications. $^{36}$ Such a variety of photonic applications emphasizes the need to understand excited-state dynamics of corrole complexes and their dependence on the nature of the metal and the peripheral substituents.

Compared to porphyrins, corroles have a contracted ring with one of the meso $\mathrm{CH}$ linking pyrrole groups missing (Scheme 1). One consequence is that the highest possible symmetry is lowered from $D_{4 h}$ to $C_{2 v}$ and the $e_{g}$ porphyrin LUMO is split into two separate orbitals. Absorption and emission spectral patterns are still similar to those of porphyrins ${ }^{37,38}$ : the Soret band occurs between 400 and $450 \mathrm{~nm}$ and, in some cases, is split in two ${ }^{39}$ because of the symmetry lowering. The Q-band (550-650 nm) is composed of two close-lying transitions $^{39,40}$ from the HOMO and HOMO-1 to the two first LUMO's and shows vibronic 
sidebands. Corroles are stronger fluorophores than porphyrins; the quantum yield of the $Q$ fluorescence increases from 0.1-0.2 in free-base corroles ${ }^{4,11,33,39,41}$ to $0.3-0.5$ and 0.76 in their Ga and Al complexes, respectively. ${ }^{5}$ Very weak fluorescence from the higher-lying Soret state(s) has been detected in free-base corroles ${ }^{8,11}$ and their Ga and Al complexes, ${ }^{42}$ but the Soret excited-state lifetimes (140-550 fs) are shorter than for many $\mathrm{Zn}$ and $\mathrm{Mg}$ porphyrins. ${ }^{43}$ The $\mathrm{Q}$ states are ultimately populated with a near-unity efficiency after Soret excitation. ${ }^{42}$

Population of the lowest $Q$ triplet state by intersystem crossing (ISC) is a crucial process, essential for singlet-oxygen sensitization and photodynamic therapy, ${ }^{24}$ as well as electroluminescence and triplet-triplet annihilation upconversion, ${ }^{44}$ but detrimental to fluorescence imaging and solar energy conversion because of fluorescence quenching and unproductive energy dissipation, respectively. The lowest Q-triplet is usually detected by transient absorption (TA) in the visible ${ }^{4,33,39,41}$ or infrared (IR) region. ${ }^{45}$ Phosphorescence at room temperature has been reported in the near-IR region for $\operatorname{Ir}^{\mathrm{III}}(\sim 790 \mathrm{~nm})^{46}$ and $\mathrm{Au} \mathrm{u}^{\mathrm{III}}(779$ $\mathrm{nm}),{ }^{47}$ as well as for iodinated $\mathrm{Al}^{\mathrm{II \prime}}, \mathrm{Ga} \mathrm{a}^{\mathrm{II}}$, and $\mathrm{P}^{\vee}(810-850 \mathrm{~nm})^{7,9}$ corrole complexes. Typically, these triplet states live hundreds of microseconds ${ }^{7,9,47}$ and undergo efficient energy-transfer quenching by oxygen. For free-base corroles, the ISC rate depends on the extent of fluorination of the meso-phenyl groups, ${ }^{8}$ increases upon $\beta$-bromination of the pyrrol rings, ${ }^{39,45}$ introducing a $\mathrm{Cl}$ or I atom on one of the meso-phenyls, ${ }^{33,41}$ or upon complexation with main-group elements Ga"I and $\mathrm{Al}^{\mathrm{III}},{ }^{42}$ as well as $\mathrm{P}^{\mathrm{V}}$ and $\mathrm{Ge}^{\mathrm{IV}}{ }^{48}$ The (near) absence of fluorescence in emission spectra of phosphorescent $\mathrm{Ir}^{\mathrm{III}}$ and $\mathrm{Au}^{\prime \prime \prime}$ complexes point to a very fast ISC, although its rate has not been reported. ${ }^{46,47}$ 
lodination of the pyrrole rings in Ga"' and All' corroles produced complexes bearing zero, three, or four $\beta$-iodine atoms (Scheme 1), further abbreviated $\mathbf{0}-\mathbf{M}, \mathbf{3}-\mathbf{M}$, and $\mathbf{4 - \mathbf { M }}$, respectively, $(M=A l, G a) .6,7$ The fluorescence yield is strongly quenched, for example from 0.76 in $\mathbf{0 - A l}$ to 0.0034 in 4-Al, while long-lived (192-373 $\mu$ s) phosphorescence emerges, ${ }^{7}$ thus indicative of fast and efficient triplet population. These compounds provide an excellent platform to investigate the physical origin of the "heavy atom effect" that is normally understood as ISC acceleration in organic compounds (usually aromatics) upon bromination, iodination, or appending sulphurcontaining groups. ${ }^{49,50,51}$ Whereas this effect is well recognized, quantitative kinetics data are rare and their origin is usually qualitatively attributed only to enhanced spin-orbit coupling (SOC), whose strength increases with the 4th power of the atomic number. Below, we show that the reality is more complex, emphasizing the need to analyze SOC pathways and effects on the whole photocycle.

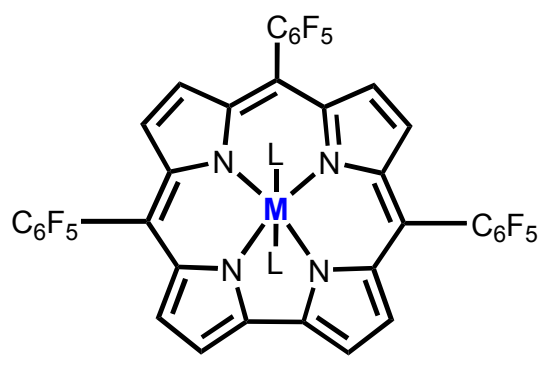

$$
\begin{array}{ll}
0-A l & \left(M=A I^{I I I}\right) \\
0-G a & \left(M=G a^{I I I}\right)
\end{array}
$$

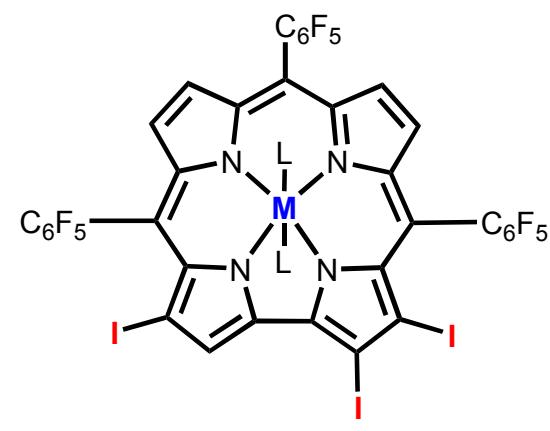

3-Ga $\quad\left(M=G a^{I I I}\right)$

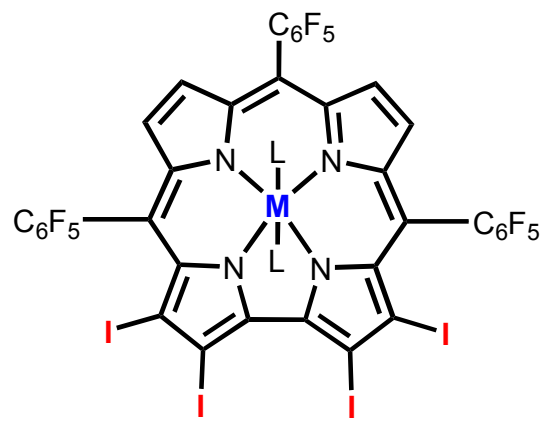

4-Al $\quad\left(\mathrm{M}=\mathrm{Al}^{\mathrm{III}}\right)$ 4-Ga $\quad\left(M=G a^{I I I}\right)$

Scheme 1. Schematic structures of investigated corrole complexes ( $L=$ pyridine).

The series of sequentially iodinated Al- and Ga-corroles provided an opportunity to investigate their photophysics using femtosecond-resolved spectrally broad-band fluorescence 
up-conversion in combination with quantum chemical calculations that explicitly included SOC. It emerged that appending iodine atom(s) at the chromophore periphery primarily introduces a manifold of low-lying charge-transfer states that provides a cascade for electronic relaxation of higher-lying Soret states and, also, opens new spin-orbit coupling pathways that mix triplet and singlet characters, facilitating ISC. At the end, all nonradiative decay processes are accelerated, whether they involve a spin-change or not.

\section{Experimental}

Materials. Corrole complexes were synthesized and characterized by literature methods. ${ }^{6,7,52,53}$ Samples for time-resolved spectroscopic studies were obtained by dissolving $20-40 \mathrm{mg}$ in $80 \mathrm{~mL}$ of toluene containing $5 \%$ of pyridine resulting in ca. $0.1-1 \mathrm{mM}$ concentration. All measurements were performed at room temperature under argon atmosphere. Spectroscopic-quality anhydrous solvents were used as obtained from Sigma-Aldrich.

Instrumentation. Steady-state absorption and emission measurements have been carried out in $1 \mathrm{~mm}$ quartz cuvettes using $1 \mathrm{mM}$ sample concentrations, to match the concentrations used in upconversion experiments. Fluorolog-3 (model FL3-11; HORIBA Jobin Yvon) and Shimadzu UV-2600 instruments were used to obtain emission and absorption spectra, respectively. Timeresolved fluorescence decays on a pico-nanosecond timescale were measured using the timecorrelated single photon counting technique (TCSPC) on an IBH $5000 \mathrm{U}$ SPC instrument equipped with a cooled Hamamatsu R3809U-50 microchannel plate photomultiplier. Samples (in a $1 \mathrm{~cm}$ cell) were excited at $405 \mathrm{~nm}$ with a Picoquant LDH-D-C-405 diode laser (50 ps fwhm) with a repetition frequency of $2.5 \mathrm{MHz}$. The detected signal was kept below 20000 counts per 
second to avoid shortening of the recorded lifetime due to the pile-up effect. Fluorescence decays were fitted (using the iterative reconvolution procedure with IBH DAS6 software) to a multiexponential function convoluted with the experimental instrument response function (IRF). Broad-band fluorescence upconversion was employed to obtain time-resolved fluorescence spectra and dynamics in the femto-picosecond range. ${ }^{54}$ Flowing sample solutions in a $0.2 \mathrm{~mm}$ cell were excited at $400 \mathrm{~nm}$ by laser pulses obtained by frequency doubling of 800 $\mathrm{nm}, 80 \mathrm{fs}$ pulses provided by a Coherent Rega Ti:Sapphire regenerative amplifier operating at $150 \mathrm{kHz}$ repetition rate. Photoluminescence was collected by a parabolic mirror in a forwardscattering geometry, and directed to a second mirror that focuses it onto a $0.25 \mathrm{~mm}$ thick $\beta$ barium borate $(\mathrm{BBO})$ crystal. The luminescence is then upconverted by mixing with the $800 \mathrm{~nm}$ gate pulse in a slightly noncollinear geometry. The up-converted signal is spatially filtered and detected with a spectrograph and a liquid-nitrogen cooled CCD camera. The kinetics of the emitted signal was measured by delaying the gate beam with respect to the onset of the fluorescence, while keeping the BBO crystal at a given angle. Polychromatic detection was enabled by rotating the BBO crystal during the accumulation time of the CCD camera, to phasematch a wide spectral region at each time delay. A $140 \mathrm{fs}$ IRF was estimated by measuring the water Raman signal, allowing us to safely determine lifetimes ca. 3-times shorter using deconvolution. Kinetics were analyzed by singular value decomposition (SVD) and global fitting. ${ }^{55}$

Quantum chemical calculations. The electronic structures of the Al and Ga metallocorroles were calculated by density functional theory (DFT) methods using the Gaussian 09.E01 (G09) ${ }^{56}$ and ADF2016.06 ${ }^{57}$ program packages. ADF was employed especially for spin-orbit calculations. 
Within G09, polarized triple $\zeta$ basis sets ${ }^{58,59,60,61}$ and hybrid B3LYP exchange and correlation functional ${ }^{62}$ were used. The solvent was described by the polarizable calculation model (PCM). ${ }^{63}$ Geometry optimizations were performed both in vacuo and with the PCM correction. The lowest singlet excited states were optimized by TDDFT methodology. Geometry optimizations were followed by vibrational analysis; no imaginary frequencies were found for the optimized structures. Electronic excitations were calculated by TDDFT at the optimized geometries. ADF calculations employed the B3LYP functional and Slater type orbital basis sets with frozen core were employed within ADF: triple- $\zeta$ quality with two polarization functions for Ga (1s-3d frozen), Al (1s-2p frozen) and I (1s-4p frozen), triple- $\zeta$ quality with one polarization function for the first row atoms (1s frozen), and double- $\zeta$ with one polarization function for $\mathrm{H}$ atoms. The scalar relativistic (SR) zero order regular approximation (ZORA) was used. Solvent effect corrections were calculated using the COSMO model. ${ }^{64}$ TDDFT calculations were performed in order to determine low-lying spin-free excited states. In the second step, spin-orbit coupling (SOC) was applied as a perturbation to obtain transition energies to spin-orbit excited states. $^{65,66}$ Calculations using PBEO and long-range corrected CAM-B3LYP functionals provided similar results to B3LYP. (CAM-B3LYP TDDFT calculated absorption spectra overestimate the Soret-band energy and the match with experimental spectra is less satisfactory than for B3LYP.)

\section{Results}

The investigated corrole complexes (Scheme 1) were synthesized as bis-pyridine adducts and investigated in toluene solutions containing $5 \%$ pyridine to stabilize the pyridine 
coordination. DFT calculations were performed on bis-pyridine adducts in toluene that was described by polarizable continuum models.

\section{DFT-calculated molecular and electronic structures}

Optimized molecular structures (Figure S1) match well experimental X-ray structures except for the calculated $\sim 16.5^{\circ}$ deviations of the iodine atoms from the corrole plane in 4-Ga and 4-Al that contrast the planar geometry found in the crystal structure, ${ }^{6}$ likely induced by crystal packing. The four spectroscopically relevant frontier MOs are shown in Figure 1 and the MO compositions are summarized in Tables S1-3. They are $\pi / \pi^{*}$ orbitals of the corrole ligand system with a negligible participation of the axial pyridines (Table S2) and they resemble the Gouterman's "four-orbital" model"67 of porphyrins. The two close-lying HOMOs are separated by ca. $0.2 \mathrm{eV}$; the metal participation is less than $0.5 \%$ and, in iodinated complexes, iodine $p(\pi)$ orbitals contribute to HOMO-1, HOMO, and LUMO by $2-10 \%$. In $\mathbf{0}-\mathbf{A l}$ and $\mathbf{0 - G a}$, the four frontier orbitals are energetically rather isolated as the HOMO-2 lies ca. $1.4 \mathrm{eV}$ lower. This changes upon iodination since a set of three predominantly (50-80\%) iodine-localized HOMO-2,3,4 (Figure S2) emerges, reducing the energy gap below HOMO-1 to 0.74 (4-Al), 0.71 (3-Ga), and $0.57 \mathrm{eV}$ (4-Ga), see Tables S1-3. lodine participation in occupied orbitals is generally larger for 4-Ga than 4-Al and 3-Ga. lodine orbitals contribute comparably to the LUMO in 4-Al and 4-Ga (less for 3-Ga) while they do not participate in LUMO+1. 


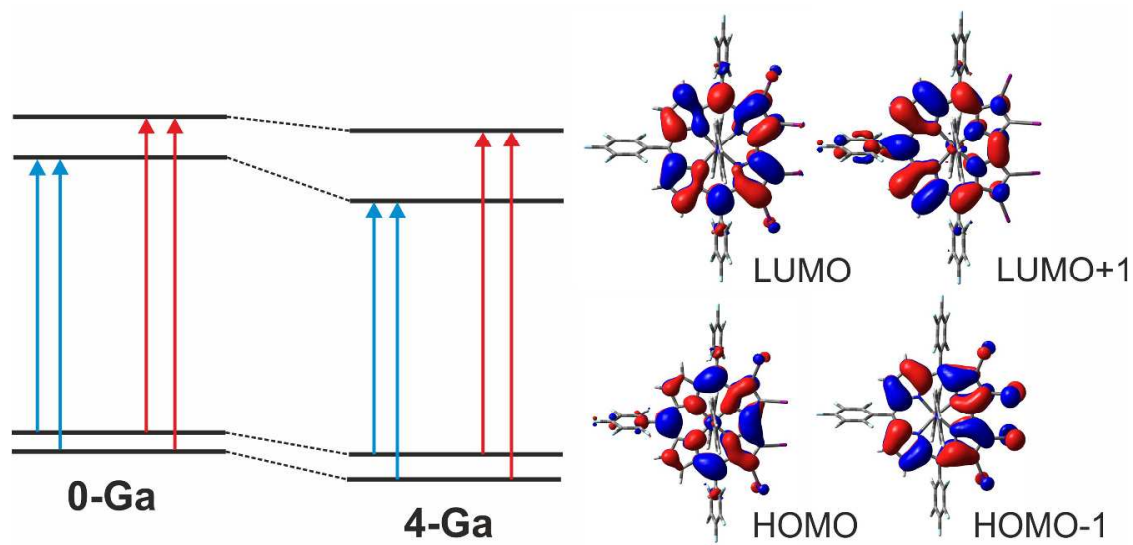

Figure 1. Left: Qualitative MO scheme of $\mathbf{0 - G a}$ and 4-Ga. Blue and red arrows correspond to the principal one-electron excitations contributing to the $Q$ and Soret transitions, respectively. Right: shapes of the corresponding MOs of $\mathbf{4 - G a . ~ ( S e e ~ r e f . ~}{ }^{37}$ for MOs of $\mathbf{0 - G a . )}$

\section{Steady-state absorption and emission spectroscopy}

All examined corroles exhibit an intense Soret absorption band between $400 \mathrm{~nm}$ and $450 \mathrm{~nm}$ and a weaker $Q$ band in the 550-650 nm range (Figures 2 and S3). ${ }^{5,6,7}$ Small differences (peak maxima shifts, occasional band splitting) between the present spectra and those published before $^{7}$ are attributable to the $5 \%$ pyridine addition used in the present study for assuring the presence of the 6-coordinated bis-pyridine adducts as the predominant species in the solution. 

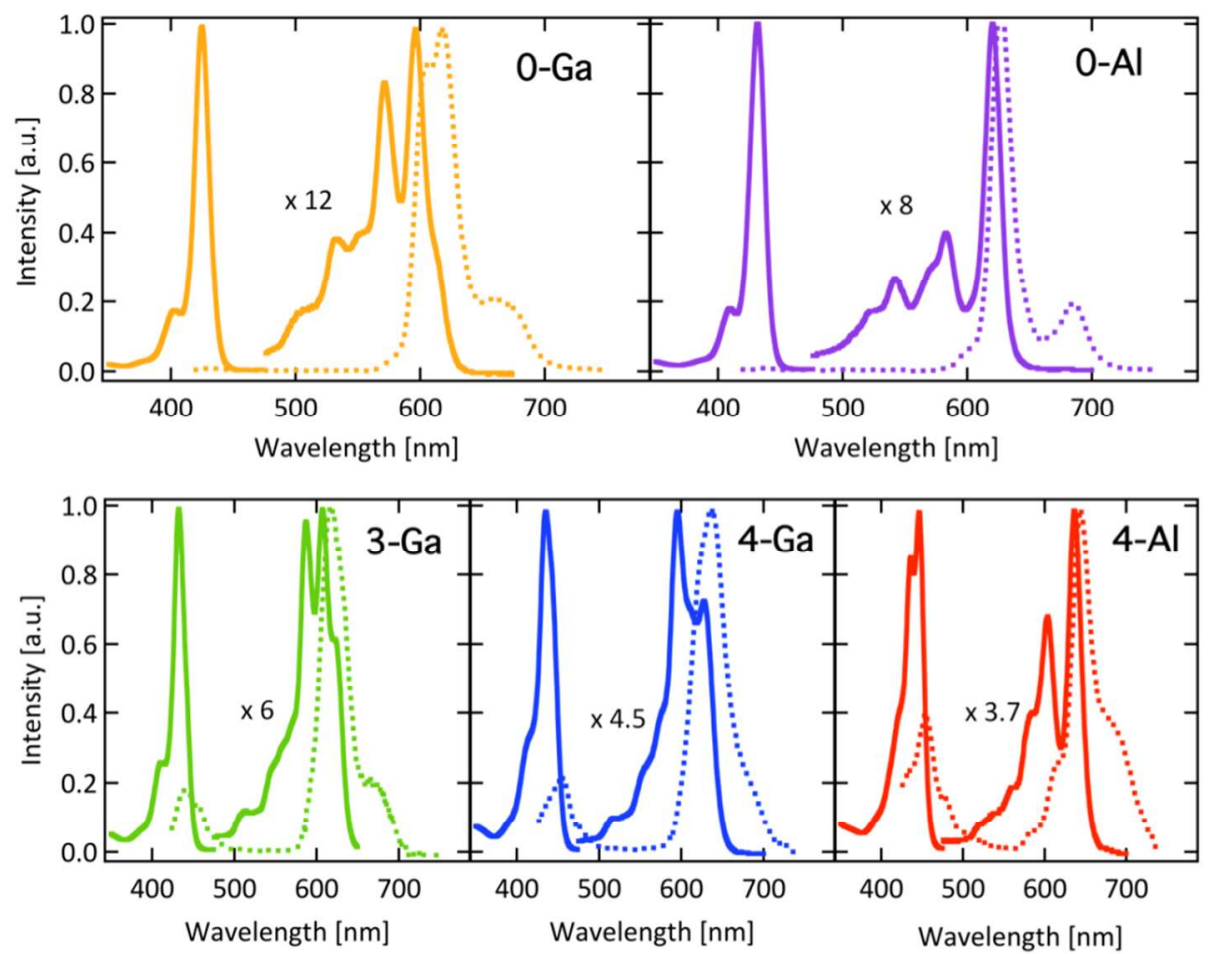

Figure 2. Normalized steady-state absorption (full) and emission spectra obtained upon $400 \mathrm{~nm}$ excitation (dashed) of the investigated complexes. Note the largely increased Q-band intensity in the iodinated derivatives, which may be appreciated by looking at the indicated multiplication factors. Molar absorptivities of the Soret and most intense $Q$ bands $\left(\mathrm{M}^{-1} \mathrm{~cm}^{-1}\right)$ : $2.94 \times 10^{5}, 3.7 \times 10^{4}\left(0-\mathrm{Al}^{53}\right) ; 2.84 \times 10^{5}, 2.36 \times 10^{4}\left(0-\mathrm{Ga}^{52}\right) ; 1.47 \times 10^{5}, 2.79 \times 10^{4}\left(3-\mathrm{Ga}^{7}\right) ; 1.48 \times 10^{5}$, $3.18 \times 10^{4}\left(4-\mathrm{Ga}^{7}\right) ; 1.16 \times 10^{5}, 3.02 \times 10^{4}\left(4-\mathrm{Al}^{6}\right)$.

TDDFT and SO-TDDFT calculations of $\mathbf{0 - G a}$ and $\mathbf{4 - G a}$ reproduce well the experimental absorption spectra, including the observed red shift and increase of the relative Q-band intensity upon iodination (Figure S4). Including SOC has a negligible effect on the calculated spectra. The Soret as well as the $\mathrm{Q}$ band each encompasses a pair of close-lying transitions of $\mathrm{A}$ and $B$ symmetry (in the $C_{2}$ point group). All these transitions arise from different combinations of one-electron excitations between the four frontier $\pi$ orbitals HOMO-1, HOMO, LUMO, and LUMO+1 (Tables S4, S5). The principal contributing excitations are indicated in Figure 1. Calculations reveal a dense manifold of states occurring between the Soret and $Q$ states of the 
iodinated complexes (Figure 3). These states involve excitations from lower-lying iodinelocalized MOs to the LUMO and possess an iodine $\rightarrow$ corrole charge transfer (CT) character. The corresponding transitions from the ground state are too weak to contribute to the absorption spectra. No such states were found for $\mathbf{0}-\mathbf{G a}$ and $\mathbf{0}$-Al where the Soret and Q pairs of states are separated by a gap of $0.7-0.8 \mathrm{eV}$.

All complexes exhibit steady-state emission in $420-480 \mathrm{~nm}$ and $500-650 \mathrm{~nm}$ ranges due the radiative decay of the Soret and Q excited states, respectively (Figures 2, S3, S5). The phosphorescence reported ${ }^{6,7}$ for the iodinated derivatives above $800 \mathrm{~nm}$ was not studied herein. By using the solvent Raman peak (Figure S5) as an internal intensity reference shows that the Soret emission of $\mathbf{0 - A l}$ and $\mathbf{0}-\mathbf{G a}$ is much stronger than for the iodinated derivatives (The Soret emission of 0-Al and 0-Ga was described before, ${ }^{42}$ but is not seen in Figures 2 and S3 because the $\mathrm{Q}$ emission of $\mathbf{0 - A l} / \mathbf{G a}$, to which the spectra are normalized, is very strong). In all cases, the Soret emission shows a weak tail towards lower energies with a shoulder at 480-500 $\mathrm{nm}$ (overlapping with the solvent Raman signal). Structured $\mathrm{Q}$ fluorescence bands are approximately mirror images of the corresponding $Q$ absorption features (Figure 2); and the presence of $5 \%$ pyridine in the toluene solvent causes small red shifts relative to the previously reported spectra. ${ }^{5,6,7,42}$ The Q-emission extends towards shorter wavelengths, which is most apparent for the iodinated complexes, namely 4-Al (Figures 2, S3). This high-energy fluorescence was attributed to hot emission from higher vibronic levels of the two $Q$ states, in analogy with the free-base ${ }^{68}$ and Zn-tetraphenylporphyrin. ${ }^{43,68}$ SO-TDDFT calculation performed at the optimized (relaxed) geometry of the $Q\left(b^{1} B\right)$ state of 4-Ga showed that the whole excitedstate manifold shifts slightly to lower energies upon excited-state relaxation (Figure S6). A good 
match between the calculated $\mathrm{Q}\left(\mathrm{a}^{1} \mathrm{~B}\right) \rightarrow \mathrm{GS}$ transition $(635 \mathrm{~nm})$ and the corresponding $0-0$ fluorescence band ( $636 \mathrm{~nm}$ in $5 \%$ pyridine/toluene; $627 \mathrm{~nm}$ in toluene) was obtained.

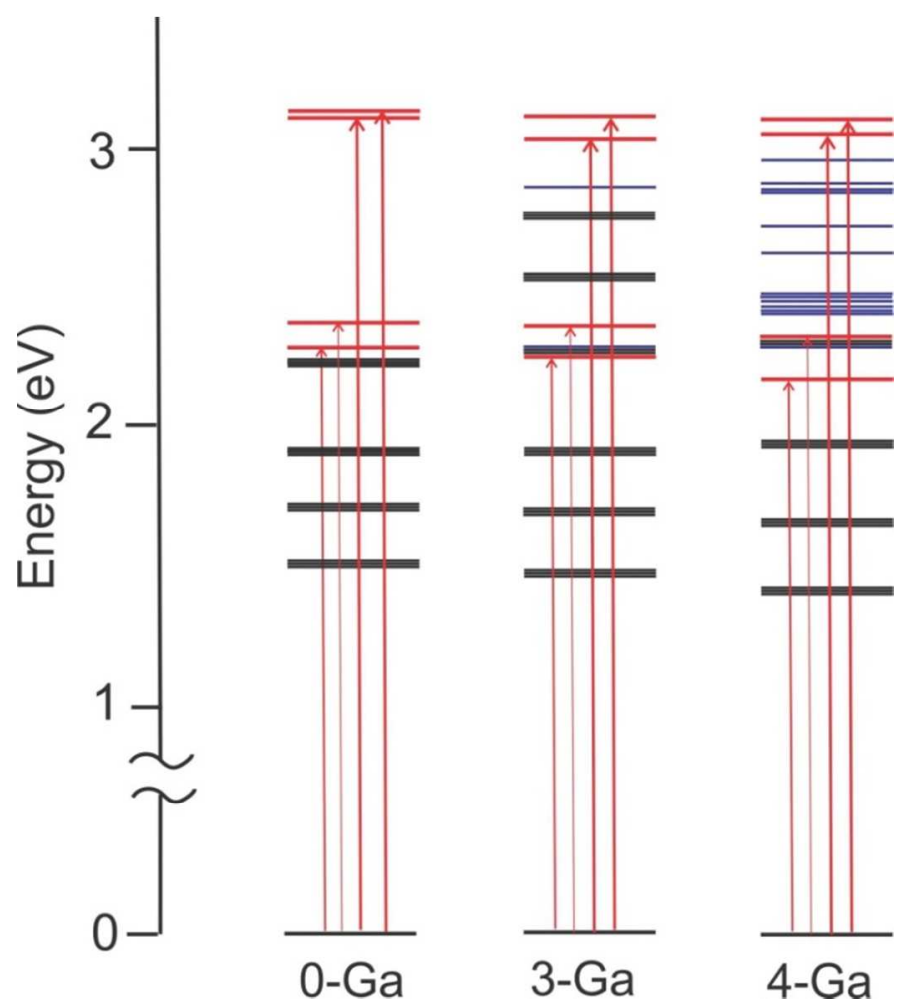

Figure 3. SO-TDDFT calculated diagram of electronic states of $\mathbf{0 - G a , ~ 3 - G a , ~ a n d ~ 4 - G a . ~ R e d ~ b a r s : ~}$ predominantly singlet states responsible for $Q$ and Soret transitions (red arrows); blue: spectroscopically weak predominantly singlet or mixed-spin states, the corresponding transitions have oscillator strengths in the range $10^{-4}-5 \times 10^{-3}$; black: predominantly triplet states.

Time-resolved fluorescence

Decay kinetics of the $\mathrm{Q}$ emission of $\mathbf{0 - A l}$ and $\mathbf{0 - G a}$ was investigated by TCSPC with ca. 80 ps time resolution. In accordance with previous data from benzene/pyridine mixtures, ${ }^{5}$ a biexponential decay was observed: 5.6 ns (81\%), 0.80 ns (19\%) for $\mathbf{0}$-Al and 2.9 ns (74\%) 0.69 ns (26\%) for $\mathbf{0}-\mathbf{G a}$. The minor components are related to kinetics of pyridine dissociation from the excited state as was discussed in detail by Kowalska et al. ${ }^{5}$ The emission decay of all iodinated 
complexes was too fast to be measured by TCSPC. Therefore, we have turned to fluorescence up-conversion with femtosecond time resolution.

Figure 4 shows the time-wavelength ( $t-\lambda)$ plots of the fs-resolved fluorescence, together with transient spectra at selected time delays. In all cases the signal at and shortly after time-zero extends over the whole spectral range and exhibits a strong feature below $500 \mathrm{~nm}$, with a clear red wing, which corresponds to the Soret emission (see Figures S5 and S7 for details of the Soret region in stationary and time-resolved spectra, resp.). The fluorescence decay in the Soret emission occurs within the first picosecond and is accompanied by a concomitant rise of the $Q$ emission at longer wavelengths $(600-650 \mathrm{~nm})$. For all iodinated complexes, the $Q$ feature is clearly observable at the earliest times after excitation (see the spectra recorded at $t=0$, Figure 4) and then grows further within the first $500 \mathrm{fs}$. Initially (until ca. $1 \mathrm{ps}$ ), the Q-emission extends to shorter wavelengths and merges with the tail of the Soret band. For 0-Al/Ga, the $Q$ feature is absent at $t=0$ and grows in the first $20 \mathrm{ps}$, on the same time scale as the Soret emission decay. At longer time delays, the $2 \mathrm{D}$ maps show that the $\mathrm{Q}$ emission is nanosecond-lived ${ }^{6,7}$ in the case of $\mathbf{0 - A l}$ and $\mathbf{0 - G a}$, whereas it is completely quenched in ca. $50 \mathrm{ps}$ in the iodinated compounds. 

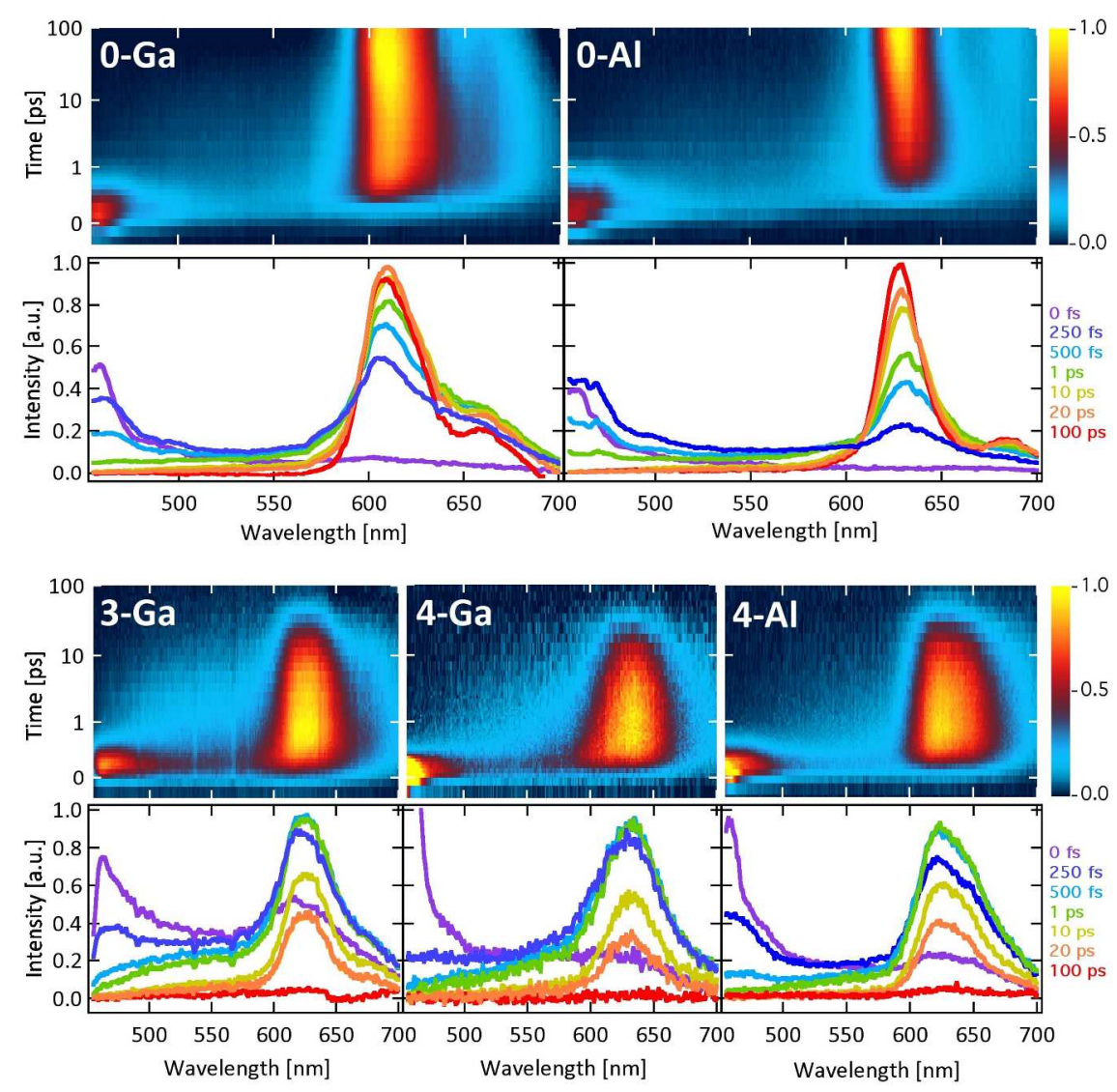

Figure 4. Fluorescence time-wavelength $2 \mathrm{D}$ plots and spectra measured at selected time delays. The time axis is displayed on a logarithmic scale, shifted by $+200 \mathrm{fs}$, in order to show also the behavior around $t=0$.

Kinetics analysis of the fluorescence time evolution was performed by Singular Value Decomposition (SVD), ${ }^{55}$ from which three time constants (Table 1) and corresponding Decay Associated Spectra (DAS, Figure 5) were extracted. SVD results are supported by a global fit (GF) analysis whereby a triexponential kinetics model was applied to kinetics traces measured at 1015 wavelengths across the spectra (Figure S8). All three time constants increase with increasing number of iodine substituents (Figure S9).

$\mathrm{DAS}_{1}$ (red) corresponding to the first sub-picosecond step $\left(\tau_{1}\right)$, is positive in the Soret emission region with a long tail all the way to the region of the $\mathrm{Q}$ emission, where it turns 
negative. This reflects the decay of the Soret- and concomitant rise of the $\mathrm{Q}$ emission. The $\tau_{1}$ time constant corresponds to the Soret-state(s) lifetime that is equal to the Q-state rise-time. For the iodinated complexes, $\mathrm{DAS}_{1}$ is also positive in the region between the Soret and $\mathrm{Q}$ features (up to $\sim 570 \mathrm{~nm}$ ), showing that the broad emission background partly decays on the $\tau_{1}$ timescale.

$\mathrm{DAS}_{2}$ resembles a second derivative of the $\mathrm{Q}$ emission band (and, possibly, of the Soret band). This is typical of band narrowing: a decay in the wings accompanied by rise of the central region around the maximum. Hence, the second kinetics step $\left(\tau_{2}\right)$ is attributable to vibrational cooling of the $\mathrm{Q}$ singlet state(s). For 3-Ga and 4-Ga and less so for 4-Al and $\mathbf{0 - G a , ~ t h e ~ p o s i t i v e ~}$ part of $\mathrm{DAS}_{2}$ extends to shorter wavelengths (up to $480-500 \mathrm{~nm}$ ) indicating that the broad emission background decays with the same kinetics. This can be understood by the fact that the Q-emission exhibits a tail extending to shorter wavelengths, which is also seen in the $\mathrm{DAS}_{3}$. The $\mathrm{DAS}_{3}$ is positive and reproduces the shape of the $\mathrm{Q}$ emission band. The respective $\tau_{3}$ lifetime thus corresponds to the decay of the $Q$ singlet excited state.

Table 1. Characteristic time constants determined by SVD analysis of the $t-\lambda$ time-resolved fluorescence up-conversion data.

\begin{tabular}{|c|c|c|c|}
\hline & $\tau_{\mathbf{1}}[\mathbf{f s}]$ & $\tau_{\mathbf{2}}$ [ps] & $\tau_{\mathbf{3}}$ [ps] \\
\hline 0-Al & $540 \pm 20$ & $16 \pm 2$ & $5600 \pm 200^{\mathrm{a}}$ \\
\hline 4-Al & $185 \pm 5$ & $1.4 \pm 0.2$ & $23.6 \pm 0.6$ \\
\hline 0-Ga & $320 \pm 20$ & $14 \pm 1$ & $2900 \pm 300^{\mathrm{a}}$ \\
\hline 3-Ga & $180 \pm 6$ & $1.4 \pm 0.1$ & $24.1 \pm 0.5$ \\
\hline 4-Ga & $70 \pm 10$ & $0.36 \pm 0.03$ & $14.9 \pm 0.6$ \\
\hline
\end{tabular}

a Determined by TCSPC. 

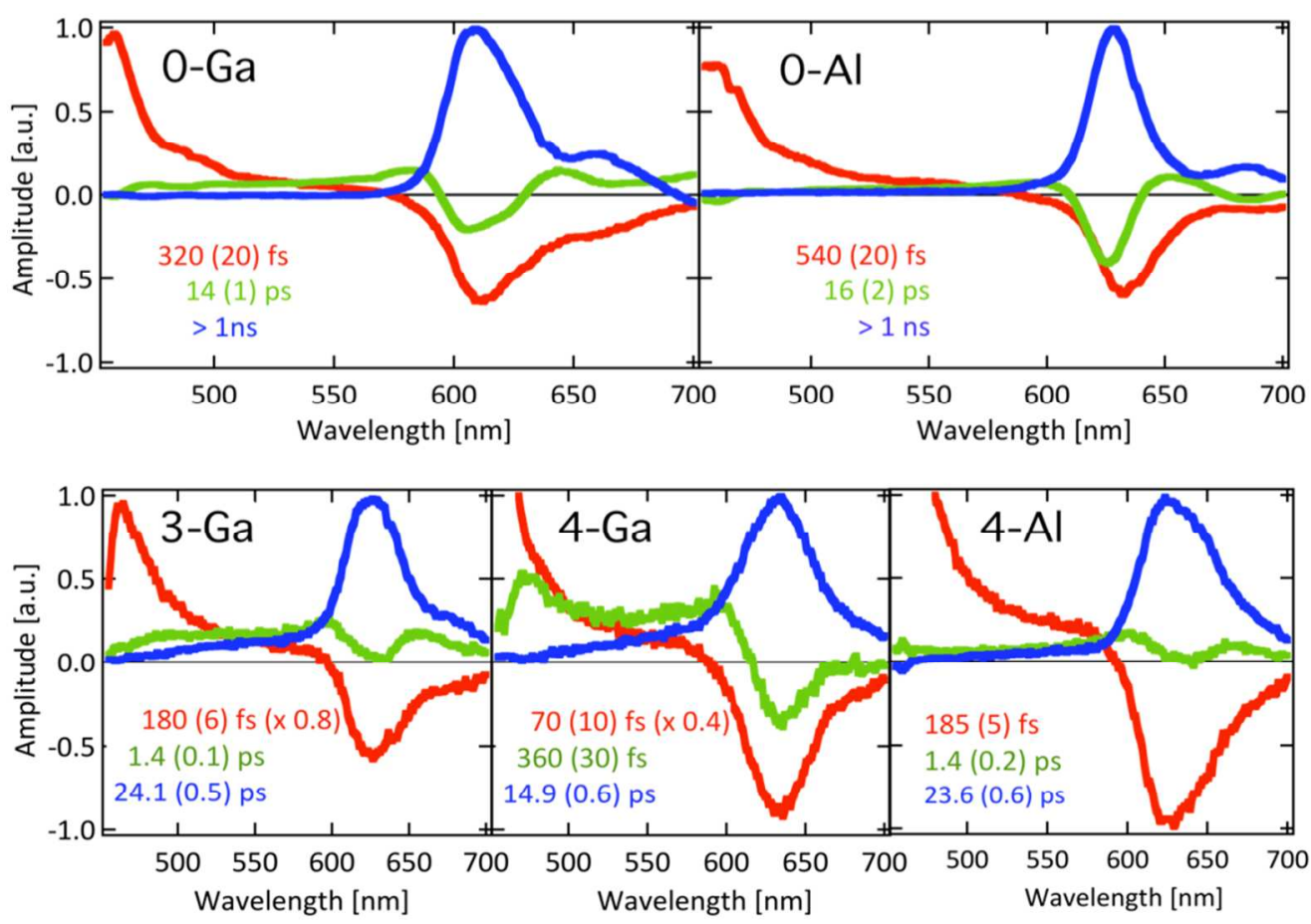

Figure 5. Decay associated spectra (DAS) obtained by SVD analysis of the $t-\lambda$ time-resolved fluorescence upconversion data. Color code: $\mathrm{DAS}_{1}$ (red); $\mathrm{DAS}_{2}$ (green); $\mathrm{DAS}_{3}$ (blue).

\section{Spin-orbit coupling pathways}

The strength of spin-orbit interactions and resulting singlet-triplet mixing were investigated by perturbational spin-orbit TDDFT calculations (SO-TDDFT). Principal SO interactions are shown in Figure 6 and summarized in Table 2. SO coupling increases with increasing number of iodine atoms, as it is indicated by the calculated values of the zero-field splitting (zfs) of the lowest triplet Q state $\mathrm{a}^{3} \mathrm{~B}: \mathbf{0}$-Ga $\left(<0.05 \mathrm{~cm}^{-1}\right)<<3-\mathbf{G a}\left(0.3 \mathrm{~cm}^{-1}\right)<\mathbf{4 - G a}(0.6$ $\left.\mathrm{cm}^{-1}\right)$; and $0.3 \mathrm{~cm}^{-1}$ for $4-\mathrm{Al}$. These values are rather low due to very small $(<0.01 \%$ for $4-\mathrm{Ga}$ ) singlet contributions to the lowest triplet. Relevant to the ISC mechanism is the spin mixing in the $Q$ singlet states. Data in Table 2 and Figure 6 show that the principal triplet admixtures to the lowest $Q$ singlet $a^{1} B$ result from SOC with the triplet charge transfer states $c^{3} B$ and $d^{3} B$ (about $0.16 \%$ each), both of which involve excitations from iodine-based MOs (Table S5). The 
higher $Q$ singlet $b^{1} A$ (HOMO- $\left.1 \rightarrow L U M O\right)$ of $4-$ Ga contains a $2.8 \%$ admixture of the $b^{3} B$ Soret triplet (HOMO-1 $\rightarrow$ LUMO+1); and this mixing is attributable to the $\sim 10 \%$ iodine contribution to HOMO-1. In addition, various ${ }^{3} \mathrm{CT}$ states introduce ca. $0.82 \%$ triplet character. Similar, albeit weaker, SO mixing occurs in 3-Ga. In 4-Al, the triplet character in the $Q$ singlet states is smaller $\left(0.06 \%\left(a^{1} B\right), 1.35 \%\left(b^{1} A\right)\right)$ than in $4-G a$ due to smaller iodine contributions to the relevant MOs (Tables S1 and S3). Singlet-triplet mixing is virtually absent in $\mathbf{0 - G a / A l ~ t h a t ~ d o ~ n o t ~ h a v e ~ a n y ~}$ intramolecular CT states.

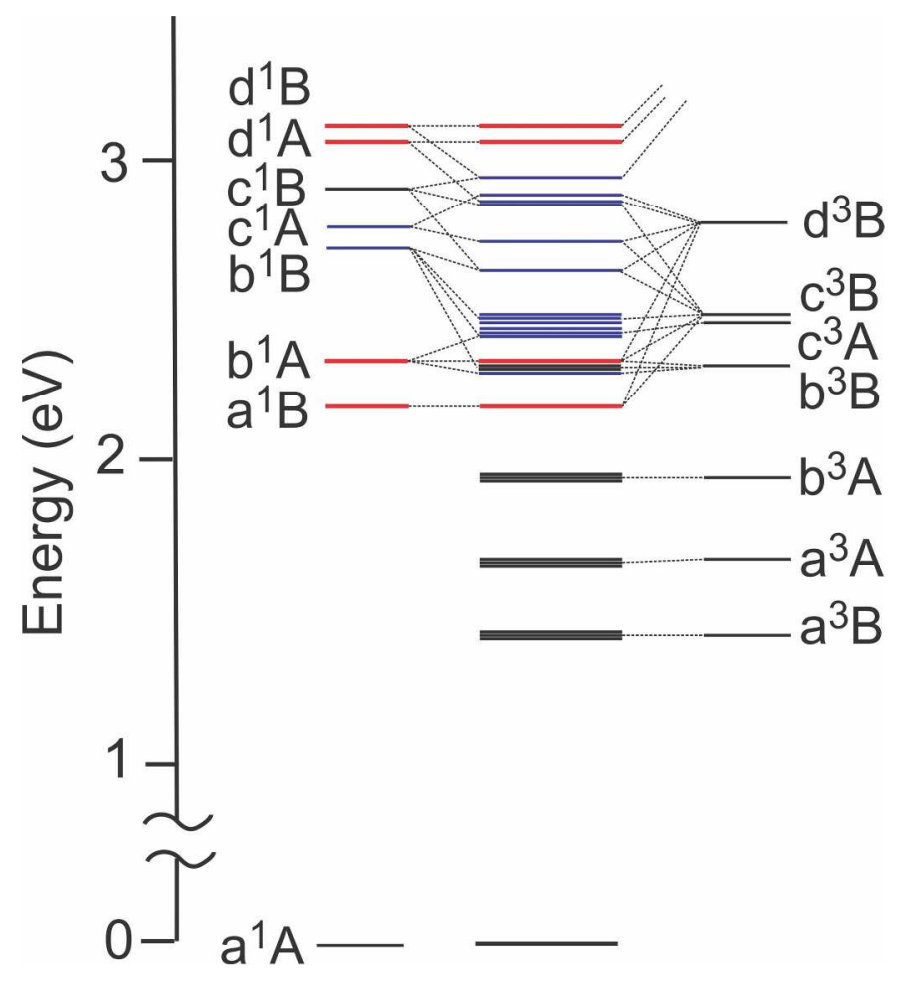

Figure 6. Principal spin-orbit interactions in 4-Ga. Left and right columns show TDDFTcalculated pure singlet and triplet states, respectively. Middle column shows the mixed-spin states calculated by SO-TDDFT. $a^{1} B$ and $b^{1} A$ are the $Q$-singlet states, $a^{3} B$ and $a^{3} A$ are the corresponding $Q$-triplets. Soret singlets are denoted $d^{1} A, d^{1} B$; and $b^{3} A, b^{3} B$ are the corresponding Soret triplets. The other states are the iodine $\rightarrow$ corrole CT states. See Table $S 5$ for contributing one-electron excitations. Color code denotes the oscillator strengths of transitions from the $\mathrm{a}^{1} \mathrm{~A}$ ground state. Red: $>0.05$, blue: 0.0001-0.05, black $<0.0001 . C_{2}$ symmetry is assumed. (The occurrence of a CT-manifold between Soret and $Q$ singlets does not 
depend on the functional used as similar results were obtained with PBEO and the long-range corrected XC functional CAM-B3LYP (Figure S10).)

Table 2. Singlet-triplet mixing in the investigated corrole complexes: zero-field splitting of the lowest $Q$ triplet; \% total admixture of triplet iodine $\rightarrow$ corrole $C T$ states to the $Q$ singlets $a^{1} B$ and $b^{1} A ; \%$ admixture of the Soret triplet $b^{3} B$ to the upper $Q$ singlet $b^{1} A$. Symmetry labels are strictly valid only for 4-Ga,Al.

\begin{tabular}{|l|c|c|c|c|}
\hline & $\mathrm{a}^{3} \mathrm{~B}$ zfs $/ \mathrm{cm}^{-1}$ & $\begin{array}{c}{ }^{3} \mathrm{CT} \text { admixture } \\
\text { to } \mathrm{a}^{1} \mathrm{~B}\end{array}$ & $\begin{array}{c}{ }^{3} \mathrm{CT} \text { admixture } \\
\text { to } \mathrm{b}^{1} \mathrm{~A}\end{array}$ & $\begin{array}{c}{ }^{3} \text { Soret admixture } \\
\text { to } \mathrm{b}^{1} \mathrm{~A}\end{array}$ \\
\hline 0-Al & $<<0.05$ & 0 & 0 & 0 \\
\hline 4-Al & 0.3 & 0.06 & 0.15 & 1.2 \\
\hline 0-Ga & $<<0.05$ & 0 & 0 & 0 \\
\hline 3-Ga & 0.2 & 0.15 & 0.10 & 0.14 \\
\hline 4-Ga & 0.6 & 0.33 & 0.82 & 2.8 \\
\hline
\end{tabular}

\section{Discussion}

Excited-state dynamics of iodine-free as well as iodinated Al and Ga corrole complexes can be described in terms of two radiative (Soret and $\mathrm{Q}$ emissions) and three nonradiative decay pathways (Scheme 2), whose mechanisms, rates and yields sensitively depend on the degree of iodination and, to a lesser extent, on the nature of the metal atom, Al vs. Ga.

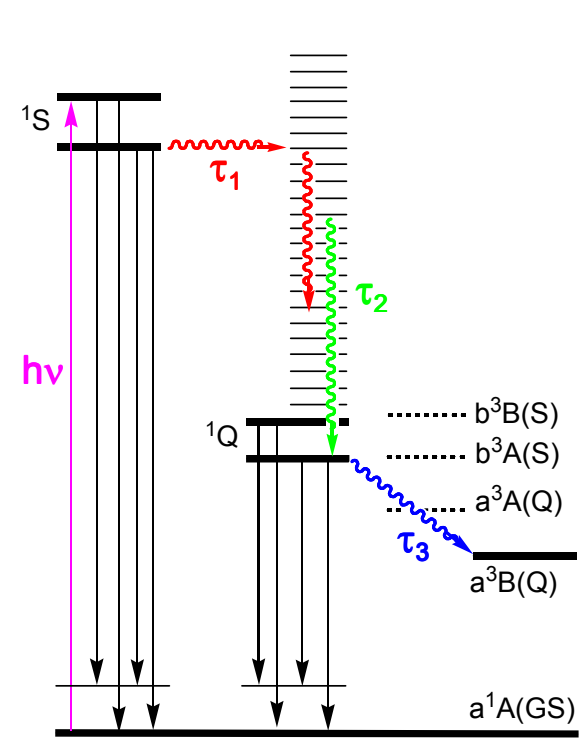

lodine-free corroles

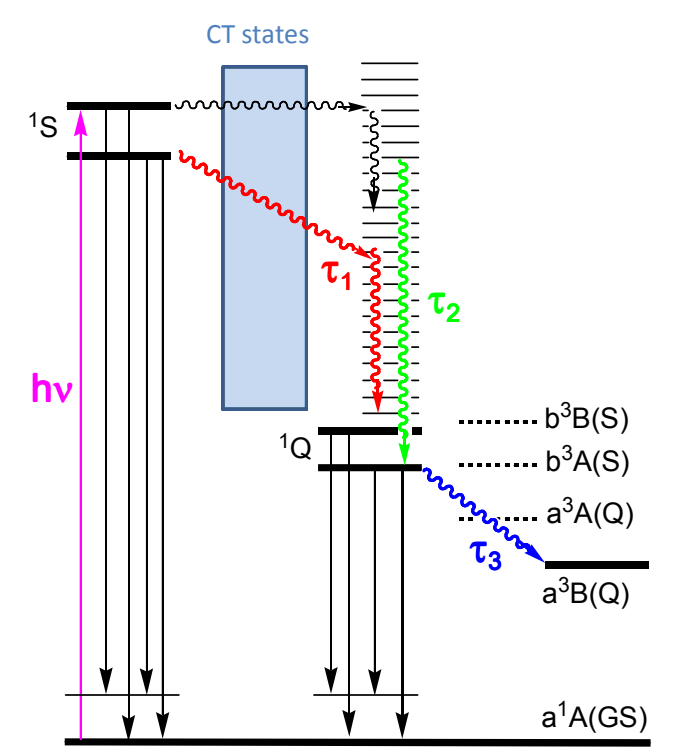

lodinated corroles 
Scheme 2. Excited-state dynamics of iodine-free (left) and iodinated (right) corrole Ga and Al complexes. Singlet Soret and $\mathrm{Q}$ pairs of states are denoted ${ }^{1} \mathrm{~S},{ }^{1} \mathrm{Q}$. The two $\mathrm{Q}$-singlets $\left({ }^{1} \mathrm{Q}\right)$ are energetically close enough to be thermally equilibrated. The arrow colors correspond to the DASs in Figure 5. Black vertical arrows denote Soret and Q fluorescence. The thin black wavy arrow in the right diagram shows the prompt energy redistribution manifested by the "instantaneous" appearance of the broad emission background. The ${ }^{1} \mathrm{~S} \rightarrow{ }^{1} \mathrm{Q}$ IC $\left(\tau_{1}\right)$ in iodinated complexes proceeds through a cascade of CT states. ISC can start from both $Q$ singlets and proceed either directly to the lowest triplet $a^{3} B(Q)$ or via $a b^{3} B(S), b^{3} A(S), a^{3} A(Q)$ cascade. It is a reversible process, as was demonstrated by observing the thermally activated delayed $Q$ fluorescence. ${ }^{7}$

Near-UV excitation populates the Soret pair of singlet excited states. This is manifested by the instantaneous appearance of the fluorescence feature at about $450 \mathrm{~nm}$, accompanied by a broad weak emission signal that extends to lower energies. For the iodinated complexes, the initial signal $(t=0)$ also exhibits a broad background around $550 \mathrm{~nm}$ due to hot emission from higher vibronic levels of the $Q\left(b^{1} A\right)$ state and a weak $Q\left(a^{1} B\right)$ emission band at $\sim 630 \mathrm{~nm}$. It follows that the Franck-Condon excited Soret state(s) is strongly coupled with higher vibronic levels of the $Q$ states and that part of the excitation energy is promptly redistributed among $Q$ vibronic and low-frequency vibrational levels within the experimental time resolution ( $<140 \mathrm{fs}$ ), concomitantly with relaxation of the Soret states. Similar ultrafast electronic and vibrational energy redistribution from higher excited states has been observed in transition metal complexes, as well as in some organic chromophores. ${ }^{54,69,70,71}$ However, this tens-offemtoseconds relaxation of Soret excited states is reserved for the iodinated corrole complexes as neither $\mathbf{0}-\mathbf{A l}$ nor $\mathbf{0}-\mathbf{G a}$ shows any signs of $\mathrm{Q}$ emission at the very early times after excitation (0-100 fs), see Figure 4.

On a longer femtosecond timescale, Soret states of non-iodinated as well as iodinated complexes undergo internal conversion (IC) to the $Q$ states, which is testified by the 
concomitant decay and rise of the Soret and Q emission bands, respectively, that occur with a common lifetime $\tau_{1}$. (The long-wavelength tail of the Soret emission decays with the same lifetime.) The $\tau_{1}$ values of $\mathbf{0}-\mathbf{A l}(540 \mathrm{fs}$ ) and $\mathbf{0 - G a}(320 \mathrm{fs}$ ) are very close to those reported earlier for 5-coordinated mono-pyridine adducts in benzene $\left(520 \pm 30,280 \pm 30 \mathrm{fs}\right.$, respectively). ${ }^{42}$ The Soret $\rightarrow Q$ conversion accelerates upon iodination to $180 \mathrm{fs}$ (3-Ga), $70 \mathrm{fs}$ (4-Ga), and $185 \mathrm{fs}$ (4Al), which is attributable to the manifold of CT excited states between Soret and $Q$ states present in the iodinated complexes (Figure 3). These states provide an energy relaxation cascade from the Soret states to various vibronic levels of the two $Q$ states. The faster IC in 4Ga relative to $\mathbf{3 - G a}$ is most likely due to the higher density of intermediate states in $\mathbf{4 - G a}$ (Figure 3). Intervening CT states likely account also for the IC acceleration on going from $\mathbf{0}$-Al (540 fs) to the $\beta$-octa-brominated complex $\mathbf{B r}_{8}-\mathbf{A l}(250 \pm 80 \mathrm{fs}),{ }^{45}$ although no computational evidence for $\mathrm{Br} \rightarrow$ corrole $\mathrm{CT}$ states was reported. On the other hand, there are no intervening electronic states in iodine- (or bromine-) free complexes and the IC takes place from relaxed Soret through high vibrational levels of the $Q$ states.

In free-base corroles, the rate of the Soret $\rightarrow$ Q IC was found ${ }^{8}$ to increase with increasing extent of fluorination of meso-phenyl rings, ranging from $(550 \mathrm{fs})^{-1}\left(F_{0}\right)$ to $(140 \mathrm{fs})^{-1}\left(F_{15}\right)$. Ge and $\mathrm{P}^{\mathrm{V}}$ corrole complexes with nonfluorinated meso-tolyl rings $\mathrm{M}(\mathrm{TTC})$ in toluene show IC time constants of 640 and $530 \mathrm{fs}$, respectively (as compared to $900 \mathrm{fs}$ for free-base TTC). ${ }^{48}$ Maingroup metal metallocorroles have shorter Soret lifetimes than analogous porphyrins ZnTPP (2.35 ps) and MgTPP (3.26 ps) in ethanol (TPP = tetraphenylporphyrin) but comparable with perfluorinated $\mathrm{Zn}\left(\mathrm{F}_{20}-\mathrm{TPP}\right)(0.46 \mathrm{ps}) .^{43}$ 
The $\tau_{2}$ kinetics correspond to vibrational cooling as indicated by the narrowing of the Qemission band. For 4-Ga, 3-Ga and (less) 4-Al and 0-Ga, the concomitant decay of the residual broad emission background indicates that the $\tau_{2}$ step also involves relaxation of higher vibronic levels of hot $b^{1} A$ and $a^{1} B Q$ states. In $\mathbf{0}-\mathbf{A l}$ (and, to some extent also $\mathbf{0 - G a}$ and $\mathbf{4 - A l}$ ), the latter process was mostly completed in the course of the Soret $\rightarrow Q$ internal conversion (i.e., on the $\tau_{1}$ timescale). The $\tau_{2}$ values are ca. 10-times shorter in the iodinated complexes than in $\mathbf{0}$-Al and $\mathbf{0}$ Ga due to intermediate states in the energetically relevant region. In addition, a better coupling with solvent modes caused by the dipolar character of the iodinated molecules and polarizable iodine atoms may play a role. The 4-fold acceleration of the $\tau_{2}$ relaxation on going from 3-Ga to 4-Ga could be attributed to the higher density of states in the latter complex. A vibrationally hot $\mathrm{Q}$ state was detected for $\mathrm{Br}_{8}$-Al by time-resolved IR spectroscopy, undergoing biexponential $\left(2,18\right.$ ps) relaxation..$^{45}$ The 2 ps component is comparable to $\tau_{2}$ of 4-Al and 3-Ga (Table 1$)$. The 18 ps one appears to be ubiquitous, present in $\mathbf{0}-\mathbf{A l}, \mathbf{0}-\mathbf{G a}, \mathbf{B r}_{\mathbf{8}}-\mathbf{A l}$, as well as in free-base corroles $^{8}$ (9-24 ps, increasing with the number of fluorine atoms on meso-Ph rings), and $M(T T C)$ (P: 6.95 ps, Ge: 13.6 ps, free-base TTC: 7.44 ps). ${ }^{48}$ It might be present also in the iodinated complexes but obscured by the ISC occurring on the same timescale.

The third kinetics component $\tau_{3}$ is due to the decay of the $Q$ singlet excited state(s): $\tau_{3}=$ $1 /\left(k_{\mathrm{r}}+k_{\mathrm{nr}}{ }^{0}+k_{\mathrm{Isc}}\right)$, where $k_{\mathrm{r}}$ and $k_{\mathrm{nr}}{ }^{0}$ account for the radiative and nonradiative decay to the ground state, respectively, and $k_{\text {ISC }}$ is the rate constant of the intersystem crossing to the lowest triplet state. The $k_{\mathrm{r}}$ and $k_{\mathrm{nr}}{ }^{0}$ values are expected to be only little dependent on the number of iodine atoms as they are not affected by SOC and the $\mathrm{I} \rightarrow$ corrole CT states occur above the $\mathrm{Q}$ singlets. For $\mathbf{0 - G a}$, values of the nonradiative decay rate constant $\left(k_{\mathrm{nr}}{ }^{0}+k_{\mathrm{ISC}}\right)$ and the triplet 
formation quantum yield $k_{\mathrm{ISC}} /\left(k_{\mathrm{nr}}{ }^{0}+k_{\mathrm{ISC}}\right)$ were both determined, ${ }^{5,33}$ providing the $k_{\mathrm{ISC}}$ value of $2.34 \times 10^{8} \mathrm{~s}^{-1}$ (i.e., $4.27 \mathrm{~ns}$ ). ${ }^{33}$ For $\mathbf{0}$-Al, the nonradiative decay rate constant of the lowest $\mathrm{Q}$ singlet state $\left(3.64 \times 10^{7} \mathrm{~s}^{-1}\right)$ is regarded as the $k_{\text {Isc }}$ upper limit since the triplet quantum yield was not reported. Strong fluorescence quenching and lifetime shortening occurs in the iodinated corrole complexes, ${ }^{6,7}$ indicating that ISC is the predominant decay process of the $Q$ singlet state(s), so that the $Q$ singlet lifetime $\tau_{3}$ can be regarded as $\sim 1 / k_{\text {Isc. }}$ Indeed, a near-unity triplet yield was determined by time-resolved IR spectroscopy for $\mathbf{B r}_{8}-\mathrm{Al}{ }^{45}$ 
Table 3. Intersystem crossing rates of corroles and their main-group complexes.

\begin{tabular}{|c|c|c|c|}
\hline & $k_{\mathrm{ISC}}\left[\mathrm{s}^{-1}\right]$ & $\tau_{\text {ISC }}[p s]$ & Remarks \\
\hline 0-Ga & $2.34 \times 10^{8}$ & 4300 & in toluene ${ }^{33}$ \\
\hline 3-Ga & $4.15 \times 10^{10}$ & 24.1 & this work \\
\hline 4-Ga & $6.71 \times 10^{10}$ & 14.9 & this work \\
\hline $0-A l$ & $<3.64 \times 10^{7}$ & $>27500$ & $a, c$ \\
\hline $0-A l$ & $<3.27 \times 10^{7}$ & $>305800$ & $b, c$ \\
\hline 4-Al & $4.24 \times 10^{10}$ & 23.6 & this work \\
\hline $\mathrm{Br}_{8}-\mathrm{Al}$ & $1.05 \times 10^{10}$ & 95 & d, ref. $^{45}$ \\
\hline \multicolumn{4}{|l|}{ free base $^{e}$} \\
\hline$F_{0}$ & $7.1 \times 10^{7}$ & 14000 & ref. $^{33}$ \\
\hline$F_{5}$ & $1.21 \times 10^{8}$ & 8260 & ref. $^{33}$ \\
\hline$F_{10}$ & $1.62 \times 10^{8}$ & 6170 & ref. $^{33}$ \\
\hline$F_{15}$ & $1.24 \times 10^{8}$ & 8070 & ref. $^{33}$ \\
\hline $\mathrm{F}_{10} / \mathrm{Cl}^{\mathrm{f}}$ & $1.28 \times 10^{8}$ & 7810 & ref. $^{33}$ \\
\hline$F_{10} / B^{f}$ & $2.14 \times 10^{8}$ & 4670 & ref. $^{33}$ \\
\hline$F_{10} / I^{f}$ & $4.69 \times 10^{8}$ & 2130 & ref. $^{33}$ \\
\hline \multicolumn{4}{|l|}{ free base $^{\mathrm{g}}$} \\
\hline no $\mathrm{Br}$ & $2.30 \times 10^{8}$ & 4350 & ref. $^{39}$ \\
\hline $\mathrm{Br}_{2}$ & $4.26 \times 10^{9}$ & 235 & ref. $^{39}$ \\
\hline $\mathrm{Br}_{3}$ & $8.10 \times 10^{9}$ & 123 & ref. $^{39}$ \\
\hline $\mathrm{Br}_{4}$ & $1.20 \times 10^{10}$ & 83 & ref. $^{39}$ \\
\hline \multicolumn{4}{|l|}{$\begin{array}{l}\text { meso-tolyl- } \\
\text {-corroles }^{\text {h }}\end{array}$} \\
\hline TTC & $3.01 \times 10^{8}$ & 3320 & ref. $^{48}$ \\
\hline $\mathrm{P}(\mathrm{TTC})(\mathrm{OH})_{2}$ & $1.09 \times 10^{9}$ & 929 & ref. $^{48}$ \\
\hline $\mathrm{Ge}(\mathrm{TTC})(\mathrm{OH})$ & $9.98 \times 10^{8}$ & 1000 & ref. $^{48}$ \\
\hline
\end{tabular}

${ }^{\mathrm{a}} k_{\mathrm{nr}}{ }^{0}+k_{\mathrm{ISC}}$ value determined in pyridine; ${ }^{5 \mathrm{~b}} k_{\mathrm{nr}}{ }^{0}+k_{\mathrm{ISC}}$ value determined in benzene; ${ }^{5 \mathrm{c}}$ Triplet formation occurs with a low quantum yield. ${ }^{72} \mathrm{~d}$ Determined by time-resolved IR spectroscopy. The lack of bleach recovery indicates $100 \%$ triplet yield. ${ }^{45}$ e tri-meso-Ph corroles with different number of $-\mathrm{C}_{6} \mathrm{~F}_{5}$ rings; $\mathrm{g}$ corroles with two trans meso- $\mathrm{C}_{6} \mathrm{~F}_{5}$ groups and one meso- $\left(p-\mathrm{C}_{6} \mathrm{H}-\right.$ ${ }_{4}(\mathrm{COOMe})$ ) substituent; ${ }^{\mathrm{h}}$ corroles with two $-\mathrm{C}_{6} \mathrm{~F}_{5}$ and one 2-hydroxy-5-halogen-phenyl meso substituents; ${ }^{\mathrm{g}}$ TTC: tri-meso-tolyl-corrole, in toluene 
The ISC rate increases with increasing number of iodine atoms $\mathbf{0}-\mathbf{G a}<<\mathbf{3 - G a}<\mathbf{4 - G a}$ and 0-Al $<<$ 4-Al (Tables 1 and 3, Figure S9). In the Ga series, the ISC rate correlates with the spinorbit coupling strength as quantified by the triplet admixture into both $\mathrm{Q}$ singlet states or by the zfs magnitude (Table 2). Qualitatively, both the SOC strength and the ISC rate can be correlated with the iodine participation in the HOMO, HOMO-1,2,3,4, and the LUMO. Analysis based on the perturbational SO-DFT calculations (Figure 6, Table 2) revealed two mechanisms whereby a triplet character is admixed to the $Q$ singlets: SO coupling of both $Q$ singlets with higher-lying iodine $\rightarrow$ corrole CT triplets (derived from HOMO-2,3,4 $\rightarrow$ LUMO excitations) and SO coupling between the higher $b^{1} A$ Q-state and the higher Soret triplet $b^{3} B$, enabled by iodine admixture to the Gouterman-like frontier orbitals HOMO-1 and, less, to HOMO and LUMO. All these SO interactions are larger for $\mathbf{4 - G a}$ than $\mathbf{3 - G a}$ and $\mathbf{4 - A l , ~ w h i c h ~ s e e m s ~ t o ~ b e ~ c a u s e d ~ b y ~}$ lower iodine participation in occupied molecular orbitals of the latter two species (Tables S1S3). Accordingly, the ISC rate in both 3-Ga and 4-Al is 1.6-times slower than in 4-Ga. The larger spin-orbit coupling constant of the heavier Ga atom as compared with Al does not have a significant effect because of the very small metal participation in the relevant MOs. The negligible metal effect is evidenced by very similar $k_{\mathrm{ISC}}$ values determined for $\mathbf{0}$-Ga and the corresponding free-base corrole. Also, ISC in $\mathrm{Ge}^{\mathrm{IV}}(\mathrm{TTC})(\mathrm{OH})$ is slightly slower than in $\mathrm{P}^{\mathrm{V}}(\mathrm{TTC})(\mathrm{OH})_{2}{ }^{48}$ (Table 3) although the opposite would be expected based on the SOC strength alone. However, the situation changes in corrole complexes with heavy transition metals such as $\operatorname{Ir}(\mathrm{III})^{46}$ or $\mathrm{Au}(\mathrm{III}),{ }^{47}$ whose $\mathrm{d} \pi$ orbitals interact with the corrole $\pi$ system (no ISC kinetics data are available for these compounds but ISC is expected to be ultrafast, based on complete fluorescence quenching). 
Similarly to $\beta$-iodo corroles, ISC is accelerated by $\beta$-bromination, as was found in $\mathbf{B r}_{\mathbf{8}}$-Al $(95 \mathrm{ps})^{45}$ and in free-base corroles, where the $\mathrm{Q}$ nonradiative decay rate increases ca. 20-, 35-, and 50 -times upon appending an increasing number $(2,3,4)$ of $\beta$-bromine atoms (Table 3). ${ }^{39}$ By analogy with iodinated corroles, bromination could also expected to introduce $\mathrm{Br} \rightarrow$ corrole $\mathrm{CT}$ states that provide SO coupling pathways. ISC in the tetrabrominated corrole is $3.5 \times$ slower than in 4-Al, which is attributable to the smaller SOC constant of $\mathrm{Br}$ as compared to I and/or to higher energies of $\mathrm{Br} \rightarrow$ corrole $\mathrm{CT}$ states.

Heavy halide atoms at meso-phenyl rings also accelerate ISC but much less than in $\beta$ positions (Table 3), due to a very small conjugation between the corrole and meso-phenyl $\pi$ systems. For example, replacing one hydrogen atom on a single $\mathrm{Ph}$ ring in a $\mathrm{Ph}\left(\mathrm{C}_{5} \mathrm{~F}_{5}\right)_{2}$-corrole by iodine accelerates the ISC $3 \times$; and $k_{\mathrm{ISC}}$ increases in the order $\mathrm{Cl}<\mathrm{Br}<1 .{ }^{33} \mathrm{~A}$ small (maximally $6 \times$ ) increase of the Q-singlet nonradiative decay rate was observed upon introducing chloro substituents on the meso-rings in meso-pyrimidinyl corroles and the effect was correlated with the total SOC coupling constant of the chloro substituents. ${ }^{41}$ Iodination also has been predicted by SO-calculations to increase SOC in Zn-phthalocyanines. ${ }^{73}$

Introducing heavy atoms into organic molecules is widely used in organic photochemistry to facilitate triplet formation. Besides corroles, the heavy atom effect has been investigated and employed in tuning the photophysics of BODIPY-type dyes. ${ }^{74}$ Introducing an iodine atom at each pyrrole ring enhanced the triplet yield and ${ }^{1} \mathrm{O}_{2}$ production. ${ }^{75,76}$ This approach was found to increase the efficiency of in vitro ${ }^{77}$ as well as in vivo ${ }^{78}$ photodynamic therapy, and to induce triplet-triplet annihilation upconversion. ${ }^{75}$ Mechanistically, the case of thiophene-derivatized BODIPY is interesting. ${ }^{79}$ The triplet yield was larger (and singlet lifetime 
shorter) when the thiophene rings were annealed to the pyrroles than if they were appended by single $\mathrm{C}-\mathrm{C}$ bonds. This difference originates from the participation of sulfur orbitals in HOMO-1 in the case of the annealed structure but not in the appended one. Consequently, SOC is stronger in the annealed derivative, leading to faster ISC. ${ }^{79}$ Along similar lines is the observation of ISC acceleration in a 4,7-bis(2-thienyl)-2,1,3-benzothiadiazole donor-acceptordonor molecule when the $\mathrm{S}$ atom(s) was(were) substituted by heavier Se with a higher SOC constant. The largest effect was observed upon replacing the $S$ atom in the acceptor (benzothiadiazole) part of the molecule with the largest $\mathrm{S} / \mathrm{Se}$ participation in the frontier orbitals (LUMO in this case). ${ }^{80}$

Molecular engineering aimed at facilitating ISC and increasing triplet yield is important when designing photosensitizers for photodynamic therapy, ${ }^{25,73}{ }^{1} \mathrm{O}_{2}$ formation, ${ }^{33,35}$ triplettriplet annihilation upconversion, ${ }^{44}$ phosphorescence sensors, ${ }^{22}$ OLED luminophores and imaging agents, as well as photoredox catalysts. ${ }^{81}$ Above, we have addressed the origins of the heavy-atom effect in $\beta$-iodinated main-group corrole complexes. In particular, we have found that changing the main-group central metal atom for a heavier one has very little effect since metal orbitals lie relatively low in energy and hardly mix with corrole orbitals. Consequently, both SOC and the density of states (DOS) are affected only a little. This becomes very different for transition metals like $\operatorname{Ir}(I I I)$ and $\mathrm{Au}(\mathrm{III})$ whose $\mathrm{d}$ orbitals interact with the corrole $\pi$ system. ${ }^{22,35,46,47}$ On the other hand, iodine atom(s) at the ligand periphery introduce CT states (increase DOS) between Soret and $Q$ singlets but not below the latter (Figures 3, 6), while iodine orbitals also participate in the corrole frontier orbitals. Both Q-singlets acquire some triplet character by mixing with the ${ }^{3} \mathrm{CT}$ states (Figure 6, Table 2), while the higher Q-singlet $b^{1} \mathrm{~A}$ 
also mixes with one of the Soret triplets $\left(b^{3} B\right)$. So, the ${ }^{3} \mathrm{CT}$ states introduced by iodination actually provide a mechanism for increasing SOC of the Q states. The CT manifold and large DOS between Soret and $\mathrm{Q}$ singlets in the iodinated complexes does not facilitate ISC from Soret singlet states: We still see distinct Q-fluorescence, which shows that ISC from Soret singlet states is inefficient (or absent) and the Q-singlet states are not bypassed in the course of Soret relaxation. In fact, the CT manifold facilitates the internal conversion between Soret and $\mathrm{Q}$ singlet states 3-5 times.

This emerging view of photophysical heavy-atom effects is applicable to a broad range of organic chromophores. Combining their extended electron-accepting $\pi$-systems with electron-rich heavy atoms (halogens, S, Se, Te) at the molecular periphery will introduce lowlying CT states, presumably with similar consequences as described herein for iodinated corroles: facilitating internal conversion and vibrational relaxation of higher singlet excited states and accelerating ISC from the lowest-lying excited singlet state into the picosecond range. Further subtle tuning of photophysical properties could be accomplished by controlling the densities and energies of the CT states.

\section{Conclusions}

Peripheral iodination of $\mathrm{Ga}$ and Al corrole complexes facilitates their nonradiative excited-state decay processes: Soret $\rightarrow Q$ internal conversion, vibrational and electronic relaxation of the two $Q$ singlet states, as well as the intersystem crossing from $Q$-singlet states to the lowest $Q$ triplet state. These photophysical "heavy-atom effects" are attributable to profound changes of the electronic structure upon iodination: (i) introducing a set of 
iodine $\rightarrow$ corrole mixed-spin charge transfer (CT) states into the energy gap between the Soret and $Q$ states, (ii) mixing of up to $10 \%$ iodine $p(\pi)$ character into the corrole frontier $\pi / \pi^{*}$ orbitals, and (iii) increasing the triplet character in the two $Q$ singlet states by enhanced spinorbit mixing with a Soret triplet and through interactions with triplet CT states.

In particular, the presence of $\mathrm{I} \rightarrow$ corrole CT states has a twofold effect on the photophysics: (i) The CT states provide a relaxation cascade for Soret $\rightarrow Q$ internal conversion, resulting in up to 5 -fold acceleration relative to the iodine-free complexes that possess a large ( $0.8 \mathrm{eV})$ Soret-Q energy gap. A higher density of intermediate states and vibrational levels in iodinated complexes also facilitates electronic and vibrational relaxation of vibronic $Q$ singlet levels. (ii) The CT states provide pathways whereby the strong iodine SOC is transmitted to the corrole $\pi$-system, resulting in up to 200 -fold acceleration of the $Q$ singlet $\rightarrow$ triplet ISC.

The peripheral heavy-atom effect in aromatic systems extends beyond facilitating ISC and enhancing phosphorescence. Dynamics of all photophysical processes are affected through increasing the density of electronic and vibrational levels, mixing the heavy-atom character into frontier MOs, and by introducing low-lying CT states that provide relaxation cascades and enhance SOC by opening new SOC pathways.

\section{- ASSOCIATED CONTENT Supporting Information}

The Supporting Information is available free of charge on the ACS Publications website at DOI: calculated $\mathbf{0 - G a}$ and $\mathbf{4 - G a}$ structures, MO shapes, energies and compositions, emission and absorption spectra, details of stationary and time-resolved Soret fluorescence, calculated absorption spectra and electronic transitions, effect of excited-state structure optimization on SO states, global fitting results.

\section{- AUTHOR INFORMATION}

Corresponding Authors 
*zalis@jh-inst.cas.cz

*majed.chergui@epfl.ch

*a.vlcek@qmul.ac.uk

\title{
Notes
}

The authors declare no competing financial interest.

\section{Acknowledgments}

This work was supported by COST Actions CM1202 and CM1405 Actions, the Czech Science Foundation (GAČR) grant 17-011375, and the Swiss NSF via the NCCR:MUST, contracts $n^{\circ}$ 200021_137717 and IZKOZ2_150425.

\author{
References
}

1. Aviv-Harel, I.; Gross, Z., Coordination chemistry of corroles with focus on main group elements. Coord. Chem. Revs 2011, 255, 717-736.

2. Aviv, I.; Gross, Z., Corrole-based applications. Chem. Commun. 2007, (20), 1987-1999.

3. Aviv-Harel, I.; Gross, Z., Aura of Corroles. Chem. - Eur. J. 2009, 15 (34), 8382-8394.

4. Ventura, B.; Degli Esposti, A.; Koszarna, B.; Gryko, D. T.; Flamigni, L., Photophysical characterization of free-base corroles, promising chromophores for light energy conversion and singlet oxygen generation. New J. Chem. 2005, 29, 1559-1566.

5. Kowalska, D.; Liu, X.; Tripathy, U.; Mahammed, A.; Gross, Z.; Hirayama, S.; Steer, R. P., Ground- and Excited-State Dynamics of Aluminum and Gallium Corroles. Inorg. Chem. 2009, 48, 2670-2676.

6. Vestfrid, J.; Botoshansky, M.; Palmer, J. H.; Durrell, A. C.; Gray, H. B.; Gross, Z., lodinated aluminum(III) corroles with long-lived triplet excited states. J. Am. Chem. Soc. 2011, 133 (33), 12899-128901.

7. Vestfrid, J.; Goldberg, I.; Gross, Z., Tuning the photophysical and redox properties of metallocorroles by iodination. Inorg. Chem. 2014, 53 (19), 10536-10542.

8. Zhang, L.; Liu, Z.-Y.; Zhan, X.; Wang, L.-L.; Wang, H.; Liu, H.-Y., Photophysical properties of electrondeficient free-base corroles bearing meso-fluorophenyl substituents. Photochem. Photobiol. Sci. 2015, 14, 953-962.

9. Vestfrid, J.; Kothari, R.; Kostenko, A.; Goldberg, I.; Tumanskii, B.; Gross, Z., Intriguing Physical and Chemical Properties of Phosphorus Corroles. Inorg. Chem. 2016, 55, 6061-6067.

10. Knyukshto, V. N.; Ngo, T. H.; Dehaen, W.; Maes, W.; Kruk, M. M., Phosphorescence of free base corroles. RSC Adv. 2016, 6, 43911-43915.

11. Ding, T.; Alemán, E. A.; Modarelli, D. A.; Ziegler, C. J., Photophysical Properties of a Series of FreeBase Corroles. J. Phys. Chem. A 2005, 109, 7411-7417.

12. Lemon, C. M.; Hwang, S. J.; Maher, A. G.; Powers, D. C.; Nocera, D. G., Halogen Photoelimination from Sb${ }^{\vee}$ Dihalide Corroles. Inorg. Chem. DOI: 10.1021/acs.inorgchem.8b00314 2018.

13. Fang, Y.; Ou, Z.; Kadish, K. M., Electrochemistry of Corroles in Nonaqueous Media. Chem. Rev. 2017, 117, 3377-3419.

14. Barata, J. F. B.; Neves, M. G. P. M. S.; Faustino, M. A. F.; Tomé, A. C.; Cavaleiro, J. A. S., Strategies for Corrole Functionalization. Chem. Rev. 2017, 117, 3192-3253.

15. Mondal, B.; Sengupta, K.; Rana, A.; Mahammed, A.; Botoshansky, M.; Dey, S. G.; Gross, Z.; Dey, A., Cobalt Corrole Catalyst for Efficient Hydrogen Evolution Reaction from $\mathrm{H}_{2} \mathrm{O}$ under Ambient Conditions: Reactivity, Spectroscopy, and Density Functional Theory Calculations. Inorg. Chem. 2013, 52, 3381-3387. 
16. Mahammed, A.; Mondal, B.; Rana, A.; Dey, A.; Gross, Z., The cobalt corrole catalyzed hydrogen evolution reaction: surprising electronic effects and characterization of key reaction intermediates. Chem. Commun. 2014, 50 (21), 2725-2727.

17. Schechter, A.; Stanevsky, M.; Mahammed, A.; Gross, Z., Four-Electron Oxygen Reduction by Brominated Cobalt Corrole. Inorg. Chem. 2012, 51, 22-24.

18. Levy, N.; Mahammed, A.; Friedman, A.; Gavriel, B.; Gross, Z.; Elbaz, L., Metallocorroles as NonPrecious Metal Electrocatalysts for Highly Efficient Oxygen Reduction in Alkaline Media. ChemCatChem 2016, 8, $2832-2837$.

19. Dogutan, D. K.; McGuire, J., R.; Nocera, D. G., Electocatalytic Water Oxidation by Cobalt(III) Hangman ß-Octafluoro Corroles. J. Am. Chem. Soc. 2011, 133, 9178-9180.

20. Gershman, Z.; Goldberg, I.; Gross, Z., DNA Binding and Catalytic Properties of Positively Charged Corroles. Angew. Chem. Int. Ed. 2007, 46, 4320-4324.

21. Gross, Z.; Golubkov, G.; Simkhovich, L., Epoxidation Catalysis by a Manganese Corrole and Isolation of an Oxomanganese(v)

Corrole. Angew. Chem. Int. Ed. 2000, 39, 4045-4047.

22. Lemon, C. M.; Powers, D. C.; Brothers, P. J.; Nocera, D. G., Gold Corroles as Near-IR Phosphors for Oxygen Sensing. Inorg. Chem. 2017, 56, 10991-10997.

23. Fischer, S.; Vestfrid, J.; Mahammed, A.; Herrmann-Westendorf, F.; Schulz, M.; Müller, J.; Kiesewetter, O.; Dietzek, B.; Gross, Z.; M., P., Photometric Detection of Nitric Oxide Using a Dissolved Iron(III) Corrole as a Sensitizer. Chempluschem 2016, 81, 594-603.

24. Mahammed, A.; Gross, Z., Corroles as Triplet Photosensitizers. Coord. Chem. Rev., http://doi.org/10.1016/j.ccr.2017.08.028.

25. Teo, R. D.; Hwang, J. Y.; Termini, J.; Gross, Z.; Gray, H. B., Fighting Cancer with Corroles. Chem. Rev. 2017, 117, 2711-2729.

26. Pribisko, M.; Palmer, J.; Grubbs, R. H.; Gray, H. B.; Termini, J.; Lim, P., Cellular uptake and anticancer activity of carboxylated gallium corroles. Proc. Natl. Acad. Sci. U.S.A. 2016, 113, E2258-E2266.

27. Agadjanian, H.; Ma, J.; Rentsendorj, A.; Valluripalli, V.; Hwang, J. Y.; Mahammed, A.; Farkas, D. L.; Gray, H. B.; Gross, Z.; Medina-Kauwe, L. K., Tumor detection and elimination by a targeted gallium corrole. Proc. Natl. Acad. Sci. USA 2009, 106, 6105-6110.

28. Blumenfeld, C. M.; Grubbs, R. H.; Moats, R. A.; Gray, H. B.; Sorasaenee, K., Decorating Metal Oxide Surfaces with Fluorescent Chlorosulfonated Corroles. Inorg. Chem. 2013, 52, 4774-4776.

29. Liang, X.; Mack, J.; Zheng, L.-M.; Shen, Z.; Kobayashi, N., Phosphorus(V)-Corrole: Synthesis, Spectroscopic Properties, Theoretical Calculations, and Potential Utility for in Vivo Applications in Living Cells. Inorg. Chem. 2014, 53, 2797-2802.

30. Walker, D.; Chappel, S.; Mahammed, A.; Brunschwig, B. S.; Winkler, J. R.; Gray, H. B.; Zaban, A.; Gross, Z., Corrole-sensitized $\mathrm{TiO}_{2}$ solar cells. J. Porphyr. Phthalocyanines 2006, 10, 1259-1262.

31. Sudhakar, K.; Giribabu, L.; Salvatori, P.; De Angelis, F., Triphenylamine-functionalized corrole sensitizers for solar-cell applications. Phys. Status Solidi A 2015, 212 (1), 194-202.

32. Luobeznova, I.; Raizman, M.; Goldberg, I.; Gross, Z., Synthesis and Full Characterization of Molybdenum and Antimony Corroles and Utilization of the Latter Complexes as Very Efficient Catalysts for Highly Selective Aerobic Oxygenation Reactions. Inorg. Chem. 2006, 45, 386-394.

33. Shao, W.; Wang, H.; He, S.; Shi, L.; Peng, K.; Lin, Y.; Zhang, L.; Ji, L.; Liu, H., Photophysical Properties and Singlet Oxygen Generation of Three Sets of Halogenated Corroles. J. Phys. Chem. B 2012, 116, 14228-14234.

34. Pohl, J.; I., S.; Mahammed, A.; Gross, Z.; Röder, B., Inhibition of green algae growth by corrole-based photosensitizers. J. Appl. Microbiol. 2015, 118, 305-312.

35. Sinha, W.; Ravotto, L.; Ceroni, P.; Kar, S., NIR-emissive iridium(III) corrole complexes as efficient singlet oxygen sensitizers. Dalton Trans. 2015, 44, 17767-17773. 
36. Anusha, P. T.; Swain, D.; Hamad, S.; Giribabu, L.; Prashant, T. S.; Tewari, S. P.; Rao, S. V., Ultrafast Excited-State Dynamics and Dispersion Studies of Third-Order Optical Nonlinearities in Novel Corroles. J. Phys. Chem. C 2012, 116, 17828-17837.

37. Rhoda, H. M.; Crandall, L. A.; Geier III, G. R.; Ziegler, C. J.; Nemykin, V. N., Combined MCD/DFT/TDDFT Study of the Electronic Structure of Axially Pyridine Coordinated Metallocorroles. Inorg. Chem. 2015, 54, 4652-4662.

38. Ziegler, C. J.; Sabin, J. R.; Geier III, G. R.; Nemykin, V. N., The first TDDFT and MCD studies of free base triarylcorroles: A closer look into solvent-dependent UV-visible absorption. Chem. Commun. 2012, 48, 4743-4745.

39. Lemon, C. M.; Halbach, R. L.; Huynh, M.; Nocera, D. G., Photophysical Properties of $\beta$-Substituted Free-Base Corroles. Inorg. Chem. 2015, 54, 2713-2725.

40. Yang, Y.; Jones, D.; von Haimberger, T.; Linke, M.; Wagnert, L.; Berg, A.; Levanon, H.; Zacarias, A.; Mahammed, A.; Gross, Z.; Heyne, K., Assignment of aluminum corroles absorption bands to electronic transitions by femtosecond polarization resolved VIS-pump IR-probe spectroscopy. J. Phys. Chem. A 2012, 116 (3), 1023-1029.

41. Nastasi, F.; Campagna, S.; Ngo, T. H.; Dehaen, W.; Maesb, W.; Kruk, M., Luminescence of mesopyrimidinylcorroles: relationship with substitution pattern and heavy atom effects. Photochem. Photobiol. Sci. 2011, 10, 143-150.

42. Liu, X.; Mahammed, A.; Tripathy, U.; Gross, Z.; Steer, R. P., Photophysics of Soret-excited tetrapyrroles in solution. III. Porphyrin analogues: Aluminum and gallium corroles. Chem. Phys. Lett. 2008, 459 (1-6), 113-118.

43. Liu, X.; Tripathy, U.; Bhosale, S. V.; Langford, S. J.; Steer, R. P., Photophysics of Soret-Excited Tetrapyrroles in Solution. II. Effects of Perdeuteration, Substituent Nature and Position, and Macrocycle Structure and Conformation in Zinc(II) Porphyrins. J. Phys. Chem. A 2008, 112, 8986-8998.

44. Singh-Rachford, T. N.; Castellano, F. N., Photon upconversion based on sensitized triplet-triplet annihilation. Coord. Chem. Rev. 2010, 254, 2560-2573

45. Stensitzki, T.; Yang, Y.; Berg, A.; Mahammed, A.; Gross, Z.; Heyne, K., Ultrafast electronic and vibrational dynamics in brominated aluminum corroles: Energy relaxation and triplet formation. Struct. Dyn. 2016, 3, 043210-043211.

46. Palmer, J. H.; Durrell, A. C.; Gross, Z.; Winkler, J. R.; Gray, H. B., Near-IR Phosphorescence of Iridium(III) Corroles at Ambient Temperature. J. Am. Chem. Soc. 2010, 132, 9230-9231.

47. Rabinovich, E.; Goldberg, I.; Gross, Z., Gold(I) and Gold(III) Corroles. Eur. J. Chem. 2011, 17, 12294 12301.

48. Raavi, S. S. K.; Yin, J.; Grancini, G.; Soci, C.; Soma, V. R.; Lanzani, G.; Giribabu, L., Femtosecond to Microsecond Dynamics of Soret-Band Excited Corroles. J. Phys. Chem. C 2015, 119, 28691-28700.

49. McClure, D. S., Triplet-Singlet Transitions in Organic Molecules. Lifetime Measurements of the Triplet State. J. Chem. Phys. 1949, 17 (10), 905-913.

50. Turro, N. J.; Ramamurthy, V.; Scaiano, J. C., Modern Molecular Photochemistry of Organic Molecules. Sausalito, California: University Science Book, 2010.

51. Solov'ev, K. N.; Borisevich, E. A., Intramolecular heavy-atom effect in the photophysics of organic molecules. Physics - Uspekhi 2005, 48 (3), 231-253.

52. Bendix, J.; Dmochowski, I. J.; Gray, H. B.; Mahammed, A.; Simkhovich, L.; Gross, Z., Structural, Electrochemical, and Photophysical Properties of Gallium(III) 5,10,15-Tris(pentafluorophenyl)corrole. Angew. Chem. Int. Ed. 2000, 39, 4048-4051.

53. Mahammed, A.; Gross, Z., Aluminum corrolin, a novel chlorophyll analogue. J. Inorg. Biochem. 2002, 88, 305-309. 
54. Cannizzo, A.; Bräm, O.; Zgrablic, G.; Tortschanoff, A.; Ajdarzadeh Oskouei, A.; van Mourik, F.; Chergui, M., Femtosecond fluorescence upconversion setup with broadband detection in the ultraviolet. Opt. Lett. 2007, 32, 3555-3557.

55. Cannizzo, A.; Blanco-Rodríguez, A. M.; Nahhas, A.; Šebera, J.; Zálišs, S.; Vlček, A., Jr.; Chergui, M., Femtosecond Fluorescence and Intersystem Crossing in Rhenium(I) Carbonyl-Bipyridine Complexes $J$. Am. Chem. Soc. 2008, 130, 8967-8974.

56. M. J. Frisch; G. W. Trucks; H. B. Schlegel; G. E. Scuseria; M. A. Robb; J. R. Cheeseman; G. Scalmani; V. Barone; B. Mennucci; G. A. Petersson; H. Nakatsuji; M. Caricato; X. Li; H. P. Hratchian; A. F. Izmaylov; J. Bloino; G. Zheng; J. L. Sonnenberg; M. Hada; M. Ehara; K. Toyota; R. Fukuda; J. Hasegawa; M. Ishida; T. Nakajima; Y. Honda; O. Kitao; H. Nakai; T. Vreven; J. A. Montgomery, J.; J. E. Peralta; F. Ogliaro; M. Bearpark; J. J. Heyd; E. Brothers; K. N. Kudin; V. N. Staroverov; R. Kobayashi; J. Normand; K. Raghavachari; A. Rendell; J. C. Burant; S. S. Iyengar; J. Tomasi; M. Cossi; N. Rega; J. M. Millam; M. Klene; J. E. Knox; J. B. Cross; V. Bakken; C. Adamo; J. Jaramillo; R. Gomperts; R. E. Stratmann; O. Yazyev; A. J. Austin; R. Cammi; C. Pomelli; J. W. Ochterski; R. L. Martin; K. Morokuma; V. G. Zakrzewski; G. A. Voth; P. Salvador; J. J. Dannenberg; S. Dapprich; A. D. Daniels; Ö. Farkas; J. B. Foresman; J. V. Ortiz; J. Cioslowski; Fox, D. J. Gaussian 09, Gaussian, Inc.: Wallingford, CT, 2009.

57. ADF2016.106. ADF2016.106, SCM, Theoretical Chemistry, Vrije Universiteit, Amsterdam, The Netherlands, http://www.scm.com.

58. Krishnan, R.; Binkley, J. S.; Seeger, R.; Pople, J. A., Self-consistent molecular orbital methods. XX. A basis set for correlated wave functions. J. Chem. Phys. 1980, 72, 650-654.

59. McLean, A. D.; Chandler, G. S., Contracted Gaussian-basis sets for molecular calculations. 1. 2nd row atoms, Z=11-18. J. Chem. Phys. 1980, 72, 5639-5648.

60. Curtiss, L. A.; McGrath, M. P.; Blaudeau, J.-P.; Davis, N. E.; Binning, R. C., Jr.; Radom, L., Extension of Gaussian-2 theory to molecules containing third-row atoms Ga-Kr. J. Chem. Phys. 1995, 103, 6104-6113. 61. Glukhovtsev, M. N.; Pross, A.; McGrath, M. P.; Radom, L., Extension of Gaussian-2 (G2) theory to bromine- and iodine-containing molecules: Use of effective core potentials. J. Chem. Phys. 1995, 103, 1878-1885.

62. Becke, A. D., Density-functional thermochemistry. III. The role of exact exchange. J. Chem. Phys. 1993, 98, 5648-5652.

63. Cossi, M.; Rega, N.; Scalmani, G.; Barone, V., Energies, Structures, and Electronic Properties of Molecules in Solution with the C-PCM Solvation Model. J. Comput. Chem. 2003, 24, 669-681.

64. Klamt, A.; Schüürmann, G., COSMO: a new approach to dielectric screening in solvents with explicit expressions for the screening energy and its gradient. J. Chem. Soc., Perkin Trans. 2 1993, 799 - 805.

65. Wang, F.; Ziegler, T., A simplified relativistic time-dependent density-functional theory formalism for the calculations of excitation energies including spin-orbit coupling effect. J. Chem. Phys. 2005, 123, 154102.

66. Wang, F.; Ziegler, T.; van Lenthe, E.; van Gisbergen, S.; Baerends, E. J., The calculation of excitation energies based on the relativistic two-component zeroth-order regular approximation and timedependent density-functional with full use of symmetry. J. Chem. Phys. 2005, 122, 204103.

67. Gouterman, G., Spectra of porphyrins. J. Mol. Spectrosc. 1961, 6, 138-163.

68. Białkowski, B.; Stepanenko, Y.; Nejbauer, M.; Radzewicz, C.; Waluk, J., The dynamics and origin of the unrelaxed fluorescence of free-base tetraphenylporphyrin. J. Photochem. Photobiol. A 2012, 234, 100106.

69. Cannizzo, A.; van Mourik, F.; Gawelda, W.; Zgrablic, G.; Bressler, C.; Chergui, M., Broadband Femtosecond Fluorescence Spectroscopy of $\left[\mathrm{Ru}(\mathrm{bpy})_{3}\right]^{2+}$. Angew. Chem. Int. Ed. 2006, 45, 3174-3176.

70. Messina, F.; Pomarico, E.; Silatani, M.; Baranoff, E.; Chergui, M., Ligand-Centred Fluorescence and Electronic Relaxation Cascade at Vibrational Time Scales in Transition-Metal Complexes. J. Phys. Chem. Lett. 2015, 6, 4475-4480. 
71. Braem, O.; Penfold, T. J.; Cannizzo, A.; Chergui, M., A femtosecond fluorescence study of vibrational relaxation and cooling dynamics of UV dyes. Phys. Chem. Chem. Phys. 2012, 14, 3513-3519.

72. Wagnert, L.; Berg, A.; Stavitski, E.; Berthold, T.; Kothe, G.; Goldberg, I.; Mahammed, A.; Simkhovich, L.; Gross, Z.; Levanon, H., Exploring the Photoexcited Triplet States of Aluminum and Tin Corroles by Time-Resolved Q-Band EPR. Appl. Magn. Reson. 2006, 30, 591-604.

73. Alberto, M. E.; De Simone, B. C.; Mazzone, G.; Sicilia, E.; Russo, N., The heavy atom effect on Zn(II) phthalocyanine derivatives: a theoretical exploration of the photophysical properties. Phys. Chem. Chem. Phys. 2015, 17, 23595-23601.

74. Yogo, T.; Urano, Y.; Ishitsuka, Y.; Maniwa, F.; Nagano, T., Highly Efficient and Photostable Photosensitizer Based on BODIPY Chromophore. J. Am. Chem. Soc. 2005, 127, 12162-12163.

75. Zhang, C.; Zhao, J.; Wu, S.; Wang, Z.; Wu, W.; Ma, J.; Guo, S.; Huang, L., Intramolecular RET Enhanced Visible Light-Absorbing Bodipy Organic Triplet Photosensitizers and Application in Photooxidation and Triplet-Triplet Annihilation Upconversion. J. Am. Chem. Soc. 2013, 135, 10566-10578.

76. Guo, S.; Ma, L.; Zhao, J.; Küçüköz, B.; Karatay, A.; Hayvali, M.; Yaglioglu, H. G.; Elmalib, A., BODIPY triads triplet photosensitizers enhanced with intramolecular resonance energy transfer (RET): broadband visible light absorption and application in photooxidation. Chem. Sci. 2014, 5, 489.

77. Gorman, A.; Killoran, J.; O'Shea, C.; Kenna, T.; Gallagher, W. M.; O'Shea, D. F., In Vitro Demonstration of the Heavy-Atom Effect for Photodynamic Therapy. J. Am. Chem. Soc. 2004, 126, 10619-10631.

78. Ma, J.; Yuan, X.; Küçüköz, B.; Li, S.; Zhang, C.; Majumdar, P.; Karatay, A.; Li, X.; Yaglioglu, H. G.; Elmali, A.; Zhao, J.; Hayvali, M., Resonance energy transfer-enhanced rhodamine-styryl Bodipy dyad triplet photosensitizers. J. Mater. Chem. C 2014, 2, 3900-3913.

79. Ji, S.; Ge, J.; Escudero, D.; Wang, Z.; Zhao, J.; Jacquemin, D., Molecular Structure-Intersystem Crossing Relationship of Heavy-Atom-Free BODIPY Triplet Photosensitizers. J. Org. Chem. 2015, 80, 5958-5963.

80. Acharya, R.; Cekli, S.; Zeman, I., C.J.; Altamimi, R. M.; Schanze, K. S., Effect of Selenium Substitution on Intersystem Crossing in $\pi$-Conjugated Donor-Acceptor-Donor Chromophores: The LUMO Matters the Most. J. Phys. Chem. Lett. 2016, 7, 693-697.

81. Sartor, S. M.; McCarthy, B. G.; Pearson, R. M.; Miyake, G. M.; Damrauer, N. H., Exploiting ChargeTransfer States for Maximizing Intersystem Crossing Yields in Organic Photoredox Catalysts. J. Am. Chem. Soc. 2018, 140, 4778-4781. 
TOC graphics

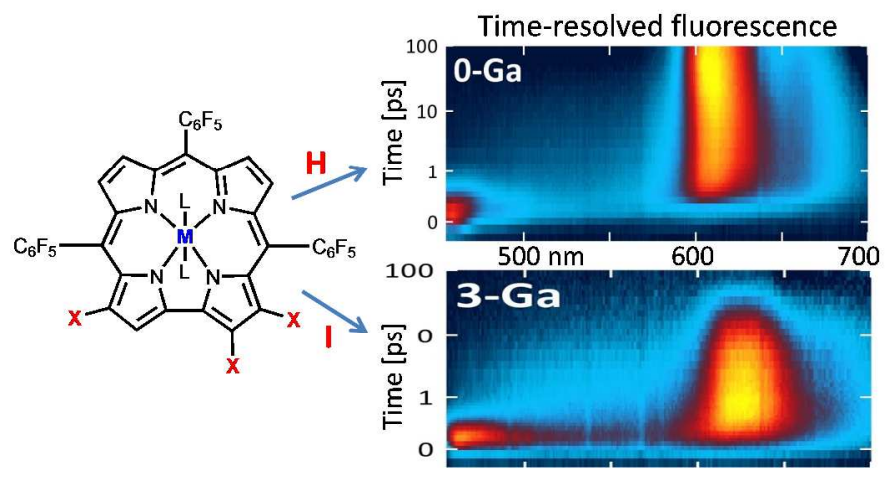

